\title{
Analysis of the modulation depth of some femtosecond laser pulses in holographic interferometry
}

\section{Halima Benzehoua}

Chouaib Doukkali University Faculty of Sciences: Universite Chouaib Doukkali Faculte des Sciences

\section{Latifa Dalil-Essakali}

Chouaib Doukkali University Faculty of Sciences: Universite Chouaib Doukkali Faculte des Sciences Abdelmajid Belafhal ( $\square$ belafhal@gmail.com)

Chouaib Doukkali University https://orcid.org/0000-0003-2735-3108

\section{Research Article}

Keywords: Femtosecond laser pulses, sh-Gaussian profile, ch-Gaussian profile, Modulation depth, Frequency detuning, Holograms

Posted Date: March 29th, 2021

DOI: https://doi.org/10.21203/rs.3.rs-281223/v1

License: (a) (1) This work is licensed under a Creative Commons Attribution 4.0 International License. Read Full License

Version of Record: A version of this preprint was published at Optical and Quantum Electronics on August 7th, 2021. See the published version at https://doi.org/10.1007/s11082-021-03133-1. 


\title{
Analysis of the modulation depth of some femtosecond laser pulses in holographic interferometry
}

\author{
H. Benzehoua, L. Dalil-Essakali, A. Belafhal* \\ Laboratory LPNAMME, Laser Physics Group, \\ Department of Physics, Faculty of Sciences, Chouaïb Doukkali University, \\ P. B 20, 24000 El Jadida, Morocco \\ *Corresponding author.E-mail: belafhal@gmail.com
}

\begin{abstract}
On the basis of the modulation depth in the interference and holographic processes, we discuss in this paper the quality of holograms generated by some femtosecond laser pulses of two different colors. The modulation depth in terms of the fringe contrast (MDFC ratio) of Higher-order sh- and ch-Gaussian temporal profiles $\left(\operatorname{sh}^{\mathrm{n}} \mathrm{GTP}\right.$ and $\left.\mathrm{ch}^{\mathrm{n}} \mathrm{GTP}\right)$ are investigated in detail. It's shown that, when we use two lasers having a very large frequency detuning, the $\mathrm{sh}^{\mathrm{n}} \mathrm{GTP}$ exhibit more precise results than the Gaussian beams for holography. $\operatorname{sh}^{\mathrm{n}} \mathrm{GTP}$ give new areas of frequency detuning to realize the very significant value of the MDFC ratio for obtaining better holograms, which is impossible with Gaussian beams. It also permits flexibility in the variable frequency difference and pulse duration for good quality holograms, and in the case of the $\operatorname{ch}^{\mathrm{n}} \mathrm{GTP}$, null regions develop and frequency bands are observed favoring the formation of the holograms. The numerical simulations are presented to illustrate and discuss the influence of the frequency detuning, the beam order and the controller parameter of the waves on the modulation depths. The propose theory will be a good basis for the development of some new experiments on the holographic interferometry and it will certainly be very useful for the specialists of the studied femtosecond laser pulses.
\end{abstract}

Keywords: Femtosecond laser pulses; sh-Gaussian profile; ch-Gaussian profile; Modulation depth; Frequency detuning; Holograms.

\section{Introduction}

In the last few decades, the dark hollow beams (DHBs) have attracted the attention of many authors because of their extensive applications in modern optics such as trapping of particles, optical communications, and guiding cold atoms [1-4]. Several models have been elaborated in the literature to describe the DHBs [5-10] by using various techniques such as the transverse mode selection [11], the optical holographic [12], the geometrical optical [13], the 
computer-generated hologram [14], and the hollow optical fibers [15] methods, to mention but a few. In 2017 Zou et al. [16] treated propagation characteristics of hollow sinh-Gaussian beams through the quadratic-index medium, and in 2021, Saad and Belafhal [17] studied the propagation of the hollow higher-order cosh-Gaussian (HhCG) beam through a quadratic index medium (QIM) and a Fractional Fourier transform (FRFT), they found that the intensity distribution of HhCG changes periodically during propagation in the QIM.

Lasers are the basic building block of the technologies for the generation of short light pulses. Shortly after the invention of this source of light, the duration of the shortest pulse produced had decreased by six orders of magnitude [18], ranging from microsecond ( $\mu \mathrm{s})$ and nanosecond (ns) regimes in the 1960s and through the picosecond (ps) regime in the seventies and finally to femtosecond (fs) techniques in the eighties [19].

On the other hand, holography is a process for recording the phase and amplitude of the wave diffracted by an object. This recording process makes it possible to later restore a threedimensional image of the object. The latter is achieved using the properties of coherent light from the laser. Holographic techniques have been a great success in various areas, such as 3D display [20] optical metrology [21], medicine [22] and many more. Lithium Niobate (LiNbO3), as photorefractive material, has proved with success its potential in being utilized in different field as holographic medium [23]. Information stored in the photorefractive material is generally erased during readout due to the dynamic nature of this material. But two color holography is a way out for non-volatile holography in which a beam of high photon energy, represented as the gating beam, is also present while recording for sensitizing the material. This beam useful in making readout non-volatile but only at larger wavelengths. A lot of Lithium Niobate systems were constructed to demonstrate non-volatile two-color holography for single hologram storage $[24,25]$. Two color hologram multiplexing was successfully studied by the use of stoichiometric [26].

Recently, recording of permanent holographic gratings using laser beams of small frequency difference has been reported by Odoulov et al. [27]. However, Malik and Escarquel [28] have reported in their work that best holograms can be produced with large frequency detuning. In 2020, Malik and Escarquel [29] showed that $\operatorname{sh}^{\mathrm{n}} \mathrm{GTP}$ for the order is equal to unity, is better than even Gaussians profile for holography, especially for higher frequencies differences. However, to the best of our knowledge, no study has treated the modulation depths of $\operatorname{sh}^{\mathrm{n}}$ GTP and $\mathrm{ch}^{\mathrm{n}} \mathrm{GTP}$ in terms of the fringe contrast. The remainder of this paper is organized as follows: The theory details for the ratio of modulation depth and fringe contrast is developed in Section 2. While Section 3 is devoted to some important numerical simulations and 
discussion of our main results applied in two general cases: $\operatorname{sh}^{\mathrm{n}} \mathrm{GTP}$ and $\operatorname{ch}^{\mathrm{n}} \mathrm{GTP}$. Finally, the calculated results are summarized in the conclusion.

\section{Theory}

In this section, we consider the interference of two femtosecond laser pulses with different amplitudes and different frequencies. We present the theory concerning the modulation depth evaluation of the considered beams. In the plane waves approximation, the two waves have the following electric fields

$E_{1}(r, t)=A_{1} f(\mathrm{t}) \cos \left(\omega_{1} t-\vec{k}_{1} \cdot \vec{r}\right)$

and

$E_{2}(r, t)=A_{2} f(\mathrm{t}) \cos \left(\omega_{2} t-\vec{k}_{2} \cdot \vec{r}\right)$,

where $A_{1}$ and $A_{2}$ are the amplitudes of the electric fields of the two lasers, $\omega_{1}$ and $\omega_{2}$ are the angular frequencies, $\vec{k}_{1}$ and $\vec{k}_{2}$ are the wave-vectors of the electric fields and $f(t)$ is their temporal profile. The intensity profiles of these beams is given by

$$
I_{1,2}(r, t)=\left|E_{1,2}(r, t)\right|^{2} \text {. }
$$

Consequently, in time and space, the intensity of the total electric field is evaluated by

$$
I(\vec{r}, t)=\left|E_{1}(\vec{r}, t)+E_{2}(\vec{r}, t)\right|^{2} .
$$

By introducing the frequency detuning of the waves $\Omega=\omega_{2}-\omega_{1}$, the difference of the wave vectors $\vec{k}=\vec{k}_{2}-\vec{k}_{1}$ and after some algebraic calculations, the intensity distribution of these two waves can be rewritten as

$$
I(\vec{r}, t)=f^{2}(t)\left[\mathrm{A}_{1}^{2}+\mathrm{A}_{2}^{2}+2 \mathrm{~A}_{1} \mathrm{~A}_{2} \cos (\Omega \mathrm{t}-\overrightarrow{\mathrm{k}} \cdot \overrightarrow{\mathrm{r}})\right]
$$

which gives a moving fringe pattern. The velocity with the pattern moves along the wave vector is evaluated by $v=\frac{\Omega}{k}$ and the fringe contrast is defined by

$$
m=\frac{2 \mathrm{~A}_{1} \mathrm{~A}_{2}}{\mathrm{~A}_{1}^{2}+\mathrm{A}_{2}^{2}}
$$

Note that the used experimental laser beams, which create the fringe pattern, are assisted with the intensity distribution given by Eq. (4). This yields a definition of the total energy density per unit area, given by

$$
\varepsilon(\overrightarrow{\mathrm{r}})=\int_{-\infty}^{+\infty} I(\vec{r}, t) d t
$$


By substituting Eq. (4) in this last equation, $\varepsilon(\overrightarrow{\mathrm{r}})$ becomes

$\varepsilon(\vec{r})=\left(\mathrm{I}_{1}+\mathrm{I}_{2}\right)+2 \sqrt{\mathrm{I}_{1} \mathrm{I}_{2}}$,

where

$\left(\mathrm{I}_{1}+\mathrm{I}_{2}\right)=\left(A_{1}^{2}+A_{2}^{2}\right) \int_{-\infty}^{+\infty} f^{2}(t) \mathrm{dt}$,

and

$\sqrt{\mathrm{I}_{1} \mathrm{I}_{2}}=A_{1} A_{2} J$

with

$J=\int_{-\infty}^{+\infty} \mathrm{dt} \cdot f^{2}(t) \cos (\Omega \mathrm{t}-\overrightarrow{\mathrm{k}} \cdot \overrightarrow{\mathrm{r}})$.

By using the Euler's formula, Eq. (10) can be written as

$J=\frac{e^{-i \overrightarrow{\mathrm{k}} \cdot \overrightarrow{\mathrm{r}}}}{2} \int_{-\infty}^{+\infty} f^{2}(t) e^{i \Omega \mathrm{t}} \mathrm{dt}+\frac{e^{i \overrightarrow{\mathrm{k}} \cdot \overrightarrow{\mathrm{r}}}}{2} \int_{-\infty}^{+\infty} f^{2}(t) e^{-i \Omega \mathrm{t}} \mathrm{dt}$,

and the total energy density per unit area becomes

$\varepsilon(\overrightarrow{\mathrm{r}})=\left(\mathrm{I}_{1}+\mathrm{I}_{2}\right)\left\{1+M_{d} \cos (\vec{k} \vec{r})\right\}$,

and this intensity can be expressed as

$\varepsilon(\overrightarrow{\mathrm{r}})=\left(A_{1}^{2}+A_{2}^{2}\right) \int_{-\infty}^{+\infty} f^{2}(t) d t\left\{1+\frac{m}{2} \frac{e^{-i k r} \int_{-\infty}^{+\infty} f^{2}(t) \mathrm{e}^{\mathrm{i} \Omega \mathrm{t}} d t+e^{i k r} \int_{-\infty}^{+\infty} f^{2}(t) \mathrm{e}^{-\mathrm{i} \Omega \mathrm{t}} d t}{\int_{-\infty}^{+\infty} f^{2}(t) d t}\right\}$.

Finally, one obtains for the modulation depth

$M_{d}=\frac{m}{2 \cos (k r)} \frac{e^{-i k r} K^{+}+e^{i k r} K^{-}}{K}$,

where

$K^{+}=\int_{-\infty}^{+\infty} f^{2}(t) \mathrm{e}^{\mathrm{i} \Omega \mathrm{t}} d t$

$K^{-}=\int_{-\infty}^{+\infty} f^{2}(t) \mathrm{e}^{-\mathrm{i} \Omega \mathrm{t}} d t$

and

$K=\int_{-\infty}^{+\infty} f^{2}(t) d t$

Eq. (14) is the main result of the present work. Note that for a Fourier transforms spectrometer, the modulation depth is also defined by Eq. (14) where $\cos (k . r)=\cos \delta$ with $\delta$ is the phase difference of the two coherent beams. If the two waves have the same frequency but 
the amplitudes are different, in this case $\Omega=0$ and Eq. (14) reduces to $M_{d}=m$. So, in this case, the modulation depth is equal to the fringe contrast and the ratio $\mathrm{MDFC} / \mathrm{m}$ is equal to unity.

In general, this MDFC ratio plays an important role in holography and holographic interferometry, because this ratio yields to the best quality of the holograms. If we have two beams, with the same amplitudes $\left(A_{1}=A_{2}=A\right)$ and the fringe contrast is equal to unity $(\mathrm{m}=1)$, $M_{d}$ is expressed as

$M_{d}=\left|\frac{1}{2 \cos (k . r)} \frac{e^{-i k r} \int_{-\infty}^{+\infty} f^{2}(t) \mathrm{e}^{\mathrm{i} \Omega \mathrm{t}} d t+e^{i k r} \int_{-\infty}^{+\infty} f^{2}(t) \mathrm{e}^{-\mathrm{i} \Omega \mathrm{t}} d t}{\int_{-\infty}^{+\infty} f^{2}(t) d t}\right|$.

In the following sub-section, we will apply our main result to two important cases: $\operatorname{sh}^{\mathrm{n}} \mathrm{GTP}$ and $\operatorname{ch}^{\mathrm{n}} \mathrm{GTP}$.

\subsection{Case of $\operatorname{sh}^{\mathrm{n}} \mathrm{GTP}$}

We apply Eq. (14) to evaluate the modulation depth of $\operatorname{sh}^{\mathrm{n}} \mathrm{GTP}$ which is given by

$$
I(t)=I_{0} \cdot f^{2}(t)=I_{0} \cdot \mathrm{e}^{-t^{2} / \tau^{2}} \cdot \operatorname{sh}^{2 \mathrm{n}}\left(\frac{\delta t}{\tau}\right)
$$

where $\tau$ is the initial pulse duration, $\mathrm{n}$ is the beam order and $\delta$ is the controller parameter of the central dark spot size of the considered pulse. $I_{0}$ is the amplitude of the profile and for simplicity, we take $I_{0}=1$.

To study the modulation depth $M_{d}^{s h^{n}}$ of $\operatorname{sh}^{\mathrm{n}} \mathrm{GTP}$, we will evaluate the terms $K, K^{+}$and $K^{-}$ given by

$$
\begin{aligned}
& K=\int_{-\infty}^{+\infty} \mathrm{e}^{-\mathrm{t}^{2} / \tau^{2}} \operatorname{sh}^{2 \mathrm{n}}\left(\frac{\delta t}{\tau}\right) d t, \\
& K^{+}=\int_{-\infty}^{+\infty} \mathrm{e}^{-\mathrm{t}^{2} / \tau^{2}} \operatorname{sh}^{2 \mathrm{n}}\left(\frac{\delta t}{\tau}\right) \mathrm{e}^{\mathrm{i} \Omega \mathrm{t}} d t,
\end{aligned}
$$

and

$$
K^{-}=\int_{-\infty}^{+\infty} \mathrm{e}^{-\mathrm{t}^{2} / \tau^{2}} \operatorname{sh}^{2 \mathrm{n}}\left(\frac{\delta t}{\tau}\right) \mathrm{e}^{-\mathrm{i} \Omega \mathrm{t}} d t
$$

To carry out these integrations, we recall the following identities [30] 
$\operatorname{sh}^{2 \mathrm{n}}(x)=\frac{(-1)^{n}}{2^{2 n}}\left\{\sum_{k=0}^{n-1}(-1)^{n-k} 2\left(\begin{array}{c}2 n \\ k\end{array}\right) \operatorname{ch}[2(\mathrm{n}-\mathrm{k}) \mathrm{x}]+\left(\begin{array}{c}2 n \\ n\end{array}\right)\right\}$,

$\int_{0}^{\infty} e^{-q^{2} t^{2}} d t=\frac{\sqrt{\pi}}{2 q}, \quad$ with $(q>0)$

and

$\int_{0}^{\infty} e^{-\beta t^{2}} \operatorname{ch}(a t) d t=\frac{1}{2} \sqrt{\frac{\pi}{\beta}}, \quad$ with $(\beta>0)$

and Eq. (18.a) becomes

$K=\frac{(-1)^{n}}{2^{2 n}} \sqrt{\pi} \tau\left\{\sum_{k=0}^{n-1}(-1)^{n-k} 2\left(\begin{array}{c}2 n \\ k\end{array}\right) e^{(n-k)^{2} \delta^{2}}+\left(\begin{array}{c}2 n \\ n\end{array}\right)\right\}$.

To calculate the integrals $K^{+}$and $K^{-}$, we consider the following expression

$R_{\gamma}=\int_{-\infty}^{+\infty} e^{-\beta t^{2}+\gamma t} s h^{2 n}(a t) d t$,

which can be written as

$R_{\gamma}=\frac{e^{\gamma^{2} / 4 \beta}}{\sqrt{\beta}} \int_{-\infty}^{+\infty} e^{-u^{2}} s h^{2 n}\left(\frac{a u}{\sqrt{\beta}}+\frac{a \gamma}{2 \beta}\right) d u$.

With the help of Eqs. (19) and (20), this last equation can be restated as

$R_{\gamma}=\frac{e^{\gamma^{2} / 4 \beta}}{\sqrt{\beta}} \frac{(-1)^{n}}{2^{2 n}}\left\{\sum_{k=0}^{n-1}(-1)^{n-k} 2\left(\begin{array}{c}2 n \\ k\end{array}\right) S_{k}+\left(\begin{array}{c}2 n \\ n\end{array}\right) \sqrt{\pi}\right\}$,

with

$S_{k}=\int_{-\infty}^{+\infty} e^{-u^{2}} \operatorname{ch}\left[2(n-k)\left(\frac{a u}{\sqrt{\beta}}+\frac{a \gamma}{2 \beta}\right)\right] d u$.

By using the identity

$\operatorname{ch}(x+y)=\operatorname{ch}(y) \operatorname{ch}(x)+\operatorname{sh}(y) \operatorname{sh}(x)$

and Eq. (21), $S_{k}$ becomes

$S_{k}=\sqrt{\pi} c h\left[(n-k) \frac{a \gamma}{\beta}\right] e^{\frac{(n-k)^{2} a^{2}}{\beta}}$.

Consequently, $R_{\gamma}$ is expressed as 


$$
R_{\gamma}=e^{\gamma^{2} / 4 \beta} \sqrt{\frac{\pi}{\beta}} \frac{(-1)^{n}}{2^{2 n}}\left\{\sum_{k=0}^{n-1}(-1)^{n-k} 2\left(\begin{array}{c}
2 n \\
k
\end{array}\right) \cdot e^{\frac{(n-k)^{2} a^{2}}{\beta}} \cdot c h\left[(n-k) \frac{a \gamma}{\beta}\right]+\left(\begin{array}{c}
2 n \\
n
\end{array}\right)\right\} .
$$

The two integrals given by Eqs. (18.b) and (18.c) can be evaluated from Eq. (29) by taking $\gamma=i \Omega$ and $\gamma=-i \Omega$, respectively. After tedious algebraic calculations, one obtains

$$
K^{+}=e^{-\Omega^{2} \tau^{2} / 4} \tau \sqrt{\pi} \frac{(-1)^{n}}{2^{2 n}}\left\{\sum_{k=0}^{n-1} 2\left(\begin{array}{c}
2 n \\
k
\end{array}\right) e^{\delta^{2}(n-k)^{2}} \cdot \operatorname{ch}[\mathrm{i} \Omega \tau \delta(n-k)]+\left(\begin{array}{c}
2 n \\
n
\end{array}\right)\right\},
$$

and

$$
K^{-}=e^{-\Omega^{2} \tau^{2} / 4} \tau \sqrt{\pi} \frac{(-1)^{n}}{2^{2 n}}\left\{\sum_{k=0}^{n-1} 2\left(\begin{array}{c}
2 n \\
k
\end{array}\right) \cdot e^{\delta^{2}(n-k)^{2}} \operatorname{ch}[-\mathrm{i} \Omega \tau \delta(n-k)]+\left(\begin{array}{c}
2 n \\
n
\end{array}\right)\right\} .
$$

Finally, for this temporal profile, the modulation depth can be rearranged as

$$
M_{d}^{s h^{n}}=m e^{-\Omega^{2} \tau^{2} / 4}\left|\frac{\sum_{k=0}^{n-1}(-1)^{n-k} 2\left(\begin{array}{c}
2 n \\
k
\end{array}\right) e^{\delta^{2}(n-k)^{2}} \cdot \cos [\Omega \tau \delta(n-k)]+\left(\begin{array}{c}
2 n \\
n
\end{array}\right)}{\sum_{k=0}^{n-1}(-1)^{n-k} 2\left(\begin{array}{c}
2 n \\
k
\end{array}\right) e^{\delta^{2}(n-k)^{2}}+\left(\begin{array}{c}
2 n \\
n
\end{array}\right)}\right| .
$$

It shows that this MDFC ratio is dependent on the frequency detuning $\Omega$ of the waves and on the parameters: the beams order $n$ and the controller parameter $\delta$, which is valid for $n \geq 1$. In the particular case for $n=1$, Eq. (32) becomes

$$
M_{d}^{s h}=m e^{-\Omega^{2} \tau^{2} / 4}\left|\frac{e^{\delta^{2}} \cos (\Omega \tau \delta)-1}{e^{\delta^{2}}-1}\right| .
$$

From this equation, one deduces the expression of the MDFC ratio of this profile with $n=1$

$$
M D F C=e^{-\Omega^{2} \tau^{2} / 4}\left|\frac{e^{\delta^{2}} \cos (\Omega \tau \delta)-1}{e^{\delta^{2}}-1}\right|,
$$

which is consistent with the well-known expression given by Malik et al [29].

\subsection{Case of $\operatorname{ch}^{\mathrm{n}} \mathrm{GTP}$}

In this case, the temporal profile is given by

$$
I(t)=I_{0} \cdot f^{2}(t)=I_{0} \cdot \mathrm{e}^{-\mathrm{t}^{2} / \tau^{2}} \operatorname{ch}^{2 \mathrm{n}}\left(\frac{\delta t}{\tau}\right)
$$

where $\delta$ is the controller parameter of the central dark spot size of the pulse, $\mathrm{n}$ is the beam order and $\tau$ is the pulse initial duration. As above, the amplitude $I_{0}$ of the profile is taken equal 
to the unity. In this section, we evaluate the integrals transforms $K, K^{+}$and $K^{-}$given by Eq. (15) corresponding to this temporal profile family. By using the following identity [30]

$\operatorname{ch}^{2 \mathrm{n}}(x)=\frac{1}{2^{2 n}}\left\{\sum_{k=0}^{n-1} 2\left(\begin{array}{c}2 n \\ k\end{array}\right) \operatorname{ch}[2(\mathrm{n}-\mathrm{k}) \mathrm{x}]+\left(\begin{array}{c}2 n \\ n\end{array}\right)\right\}$,

and with the help of Eq. (20), the first integral becomes

$K=\frac{\sqrt{\pi} \tau}{2^{2 n}}\left\{\sum_{k=0}^{n-1} 2\left(\begin{array}{c}2 n \\ k\end{array}\right) e^{(n-k)^{2} \delta^{2}}+\left(\begin{array}{c}2 n \\ n\end{array}\right)\right\}$.

For the evaluation of $K^{+}$and $K^{-}$, we determine the integral transforms

$R_{\gamma}^{\prime}=\int_{-\infty}^{+\infty} e^{-\beta t^{2}+\gamma t} \operatorname{ch}^{2 n}(a t) d t$

which can be written, if one uses Eq. (36) and the following change of variable: $x=\frac{a}{\sqrt{\beta}} u+\frac{a}{2 \beta} \gamma$, as

$R_{\gamma}^{\prime}=\frac{e^{\gamma^{2} / 4 \beta}}{\sqrt{\beta}} \frac{1}{2^{2 n}}\left\{\sum_{k=0}^{n-1} 2\left(\begin{array}{c}2 n \\ k\end{array}\right) S_{k}+\left(\begin{array}{c}2 n \\ n\end{array}\right) \sqrt{\pi}\right\}$.

By using of the expression of $S_{k}$ given by Eq. (26), $R_{\gamma}^{\prime}$ becomes

$R_{\gamma}^{\prime}=e^{\gamma^{2} / 4 \beta} \sqrt{\frac{\pi}{\beta}} \frac{1}{2^{2 n}}\left\{\sum_{k=0}^{n-1} 2\left(\begin{array}{c}2 n \\ k\end{array}\right) \cdot e^{\frac{(n-k)^{2} a^{2}}{\beta}} \cdot \operatorname{ch}\left[(n-k) \frac{a \gamma}{\beta}\right]+\left(\begin{array}{c}2 n \\ n\end{array}\right)\right\}$.

The integrals transforms $K^{+}$and $K^{-}$correspond to the value of $R_{\gamma}^{\prime}$ for $\gamma=i \Omega$ and $\gamma=-i \Omega$, respectively. Consequently, one obtains

$$
K^{+}=e^{-\Omega^{2} \tau^{2} / 4} \tau \sqrt{\pi} \frac{1}{2^{2 n}}\left\{\sum_{k=0}^{n-1} 2\left(\begin{array}{c}
2 n \\
k
\end{array}\right) \cdot e^{\delta^{2}(n-k)^{2}} \cdot \operatorname{ch}[\mathrm{i} \Omega \tau \delta(n-k)]+\left(\begin{array}{c}
2 n \\
n
\end{array}\right)\right\},
$$

and

$$
K^{-}=e^{-\Omega^{2} \tau^{2} / 4} \tau \sqrt{\pi} \frac{1}{2^{2 n}}\left\{\sum_{k=0}^{n-1} 2\left(\begin{array}{c}
2 n \\
k
\end{array}\right) \cdot e^{\delta^{2}(n-k)^{2}} \cdot \operatorname{ch}[-\mathrm{i} \Omega \tau \delta(n-k)]+\left(\begin{array}{c}
2 n \\
n
\end{array}\right)\right\} .
$$

With the help of the previous results, and in the case of $\operatorname{ch}^{\mathrm{n}} \mathrm{GTP}$, Eq. (14) can be written as

$$
M_{d}^{c h^{n}}=m e^{-\Omega^{2} \tau^{2} / 4}\left|\frac{\sum_{k=0}^{n-1} 2\left(\begin{array}{c}
2 n \\
k
\end{array}\right) \cdot e^{\delta^{2}(n-k)^{2}} \cdot \cos [\Omega \tau \delta(n-k)]+\left(\begin{array}{c}
2 n \\
n
\end{array}\right)}{\sum_{k=0}^{n-1} 2\left(\begin{array}{c}
2 n \\
k
\end{array}\right) e^{\delta^{2}(n-k)^{2}}+\left(\begin{array}{c}
2 n \\
n
\end{array}\right)}\right| .
$$


It proves that the MDFC ratio is dependent for this temporal profile on the frequency detuning $\Omega$ of the waves and on the two parameters: the beams order $\mathrm{n}$ and the controller parameter $\delta$. One notes that Eq. (43) is the closed-form of the modulation depth of $\operatorname{ch}^{\mathrm{n}} \mathrm{GTP}$ and for the first order $n=1$ of this beams family, one finds the following expression of the modulation depth rearranged as

$$
M_{d}^{c h}=m e^{-\Omega^{2} \tau^{2} / 4}\left|\frac{e^{\delta^{2}} \cdot \cos (\Omega \tau \delta)+1}{e^{\delta^{2}}+1}\right| .
$$

From Eqs. (33) and (44), one deduces the MDFC ratio of a Gaussian profile as follows

$$
M D F C^{G}=e^{-\Omega^{2} \tau^{2} / 4},
$$

which is consistent with the-known expression for a Gaussian profile [25].

\section{Numerical analysis and discussions}

In this section, we will present some numerical simulations to study the dependence of the MDFC ratio on the frequency detuning for $\operatorname{sh}^{\mathrm{n}} \mathrm{GTP}, \mathrm{ch}^{\mathrm{n}} \mathrm{GTP}$ and especially for the Gaussian profiles. The present analysis is based on the analytical expressions given by Eqs. (32), (43) and (45).

\subsection{Case of Gaussian temporal profile}

In this sub-section, we illustrate in Fig. 1 the MDFC ratio for the Gaussian pulses given by Eq. (45) in terms of the pulse duration $\tau$ and the frequency detuning $\Omega$ for (a) threedimensional form and (b) in tree-dimensional (x-y) plot.

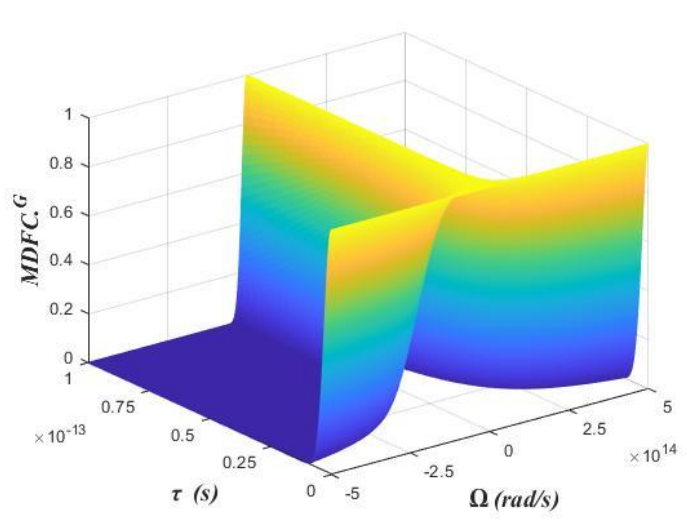

(a)

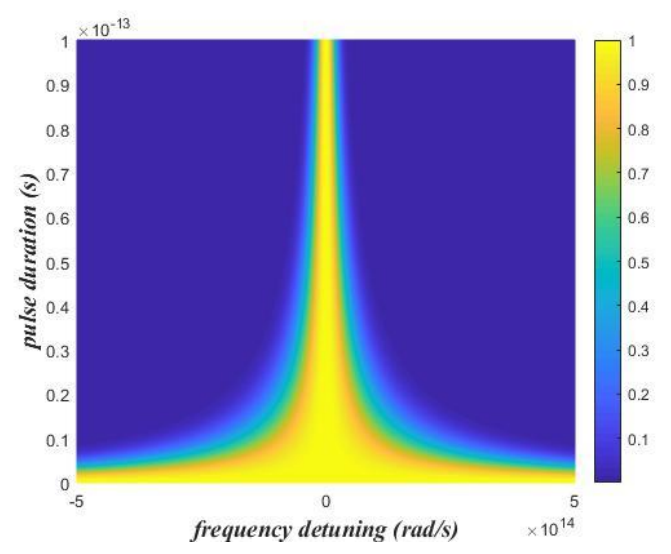

(b)

Figure 1: The MDFC ratio as a function of the pulse duration $\tau$ and frequency detuning $\Omega$ of the Gaussian pulses. 
From this figure, one observes that the MDFC ratio increases from 0 to 1 as the color changes from blue to yellow (see the rectangular bar). It can be seen also that the value of the MDFC ratio decreases when the pulse duration increases and the frequency detuning is larger for the Gaussian profile. Consequently, the appropriate value of the MDFC is obtained for a higher pulse duration and a small frequency difference.

\subsection{Case of $\operatorname{sh}^{\mathrm{n}} \mathrm{GTP}$}

We investigate here the MDFC ratio of the temporal profile given by Eq. (32). We illustrate in Fig. 2 the temporal profile of $\operatorname{sh}^{\mathrm{n}}$ GTP expressed by Eq. (17) for two values of the beam order $n$ and by varying the controlled parameter $\delta$.

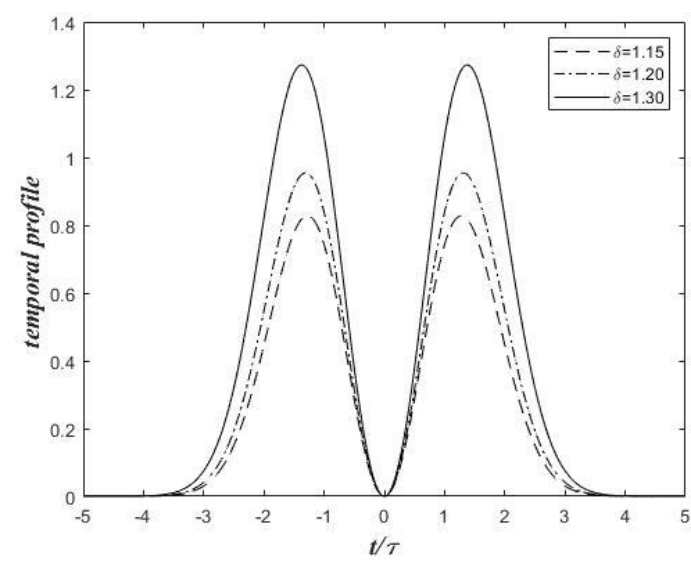

(a)

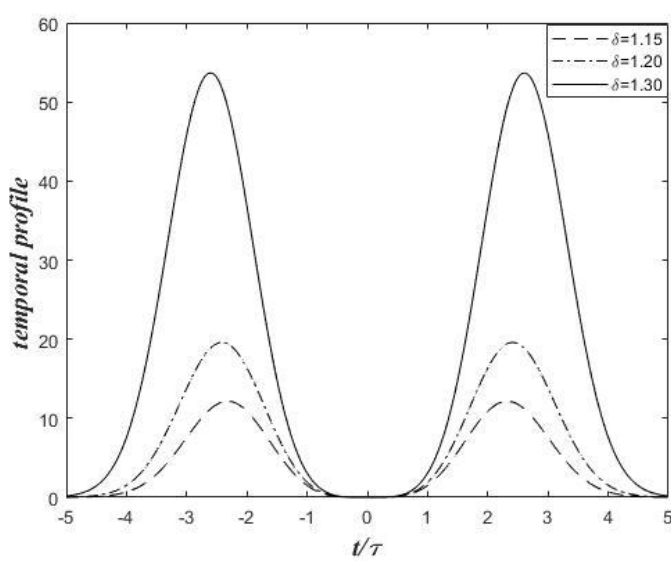

(b)

Figure 2: Temporal profile of $\operatorname{sh}^{\mathrm{n}} \mathrm{GTP}$

for different skew parameters $\delta$ and for: (a) $n=1$ and (b) $n=2$.

As it's shown from this figure, the central dark spot of this dark hollow temporal profile widens with the increase of the beam order $n$, and the decrease of the parameter $\delta$. We also observe that the intensity of the considered profile takes a large value with the increment of the two parameters $n$ and $\delta$.

From our main result represented by Eq. (32), we investigate some numerical examples for the present case. For that we illustrate in Fig. 3 the variation of MDFC ratio with the frequency detuning for different values of $\delta(0.1,0.5$, and 1.3). One observes from this figure that the width of the lobes becomes larger as the value of $\delta$ is small, and new secondary lobes appear when the beam order $n$ increases. We present in Fig. 4 the variation of the MDFC ratio with the frequency detuning for different values of the pulse duration and for a fixed value of the skew parameter $\delta(\delta=0.1)$. We can see clearly the effect of the beam order $\mathrm{n}$ on the MDFC 
ratio, which results in the appearance of new secondary lobes when $\mathrm{n}$ increases. The parameter $\tau$ has also an influence on the width of the principal lobe as well as that of the secondary lobes.

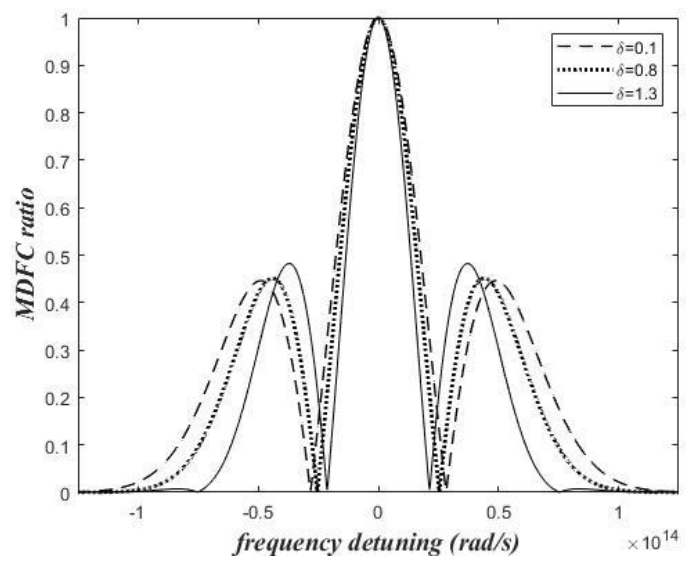

(a)

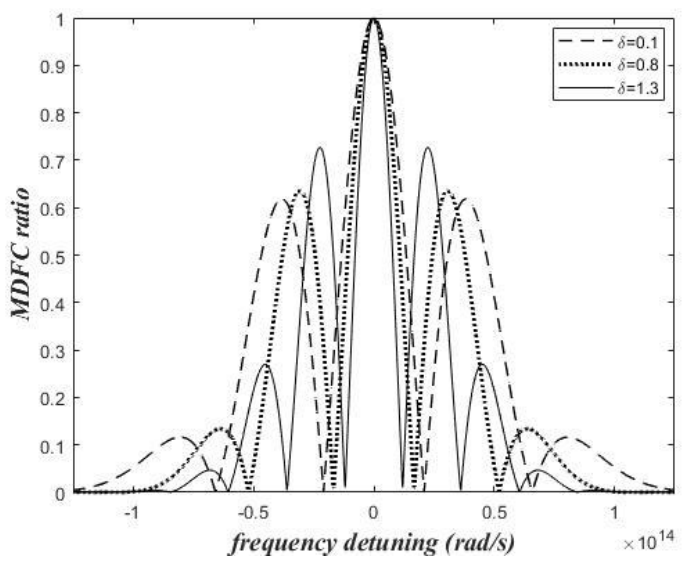

(b)

Figure 3: Behaviour of the MDFC ratio of $\operatorname{sh}^{\mathrm{n}} \mathrm{GTP}$ as a function of the frequency detuning and for a fixed value of the pulse duration set as $\tau=50 f s$ and for: (a) $n=1$ and (b) $n=2$.

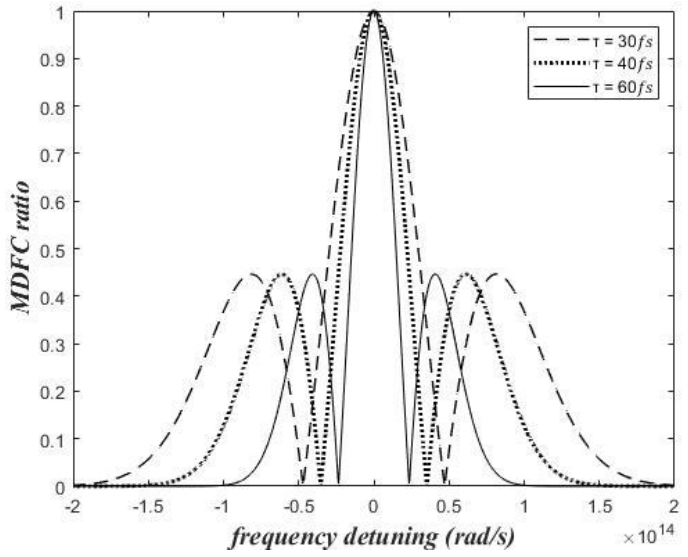

(a)

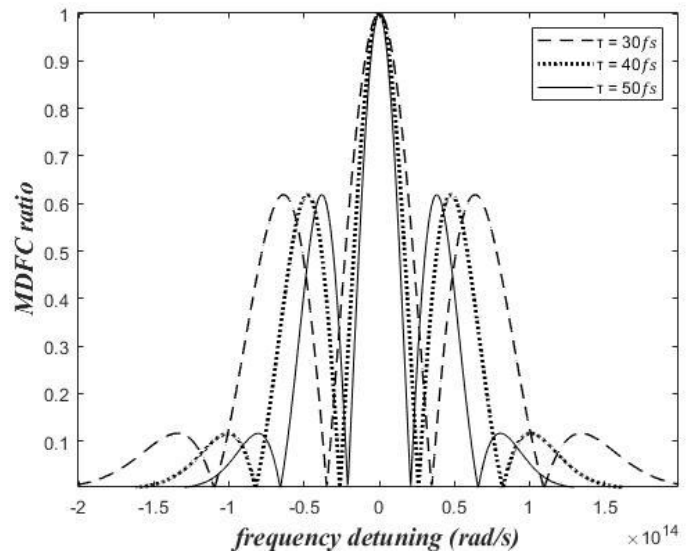

(b)

Figure 4: Plots of the MDFC ratio of $\operatorname{sh}^{n}$ GTP with the frequency detuning for: (a) $n=1$ and (b) $n=2$.

To show the effects of the beam order $\mathrm{n}$ and the skew parameters $\delta$, we illustrate in Fig. 5 the MDFC ratio as a function of the frequency detuning for the $\operatorname{sh}^{\mathrm{n}} \mathrm{GTP}$ (dashed curve) and the Gaussian profile (solid curve) for four values of $\mathrm{n}$ and two values of $\delta$. It's shown from these plots that for a particular frequency detuning, the MDFC ratio of $\operatorname{sh}^{\mathrm{n}} \mathrm{GTP}$ is higher than the MDFC ratio of the Gaussian profile (red hatched area). For example, the MDFC ratio has a value of 0.45 in the case of $\operatorname{sh}^{\mathrm{n}}$ GTP when $n=1$ (Fig. 5 (a)), which is much greater than the obtained one with Gaussian profile (i.e. MDFC ratio $=0.29$ ); this is more pronounced when the controlled parameter is smaller (i.e. $\delta=0.1$ ). We can also observe side lobes that take place when the beam order increases. It is clear that the total number of the secondary lobes is proportional to the considered profile order. For example, for $n=1$, we find only one secondary 
lobe but when $n=3$, we note three lobes and when $n=4$, another lobe starts to rise (four secondary lobes). We note that when the controlled parameter and the beam order are equal to unity, the MDFC ratio is identical to that one investigated by Malik and Escarquel [28].

(a)
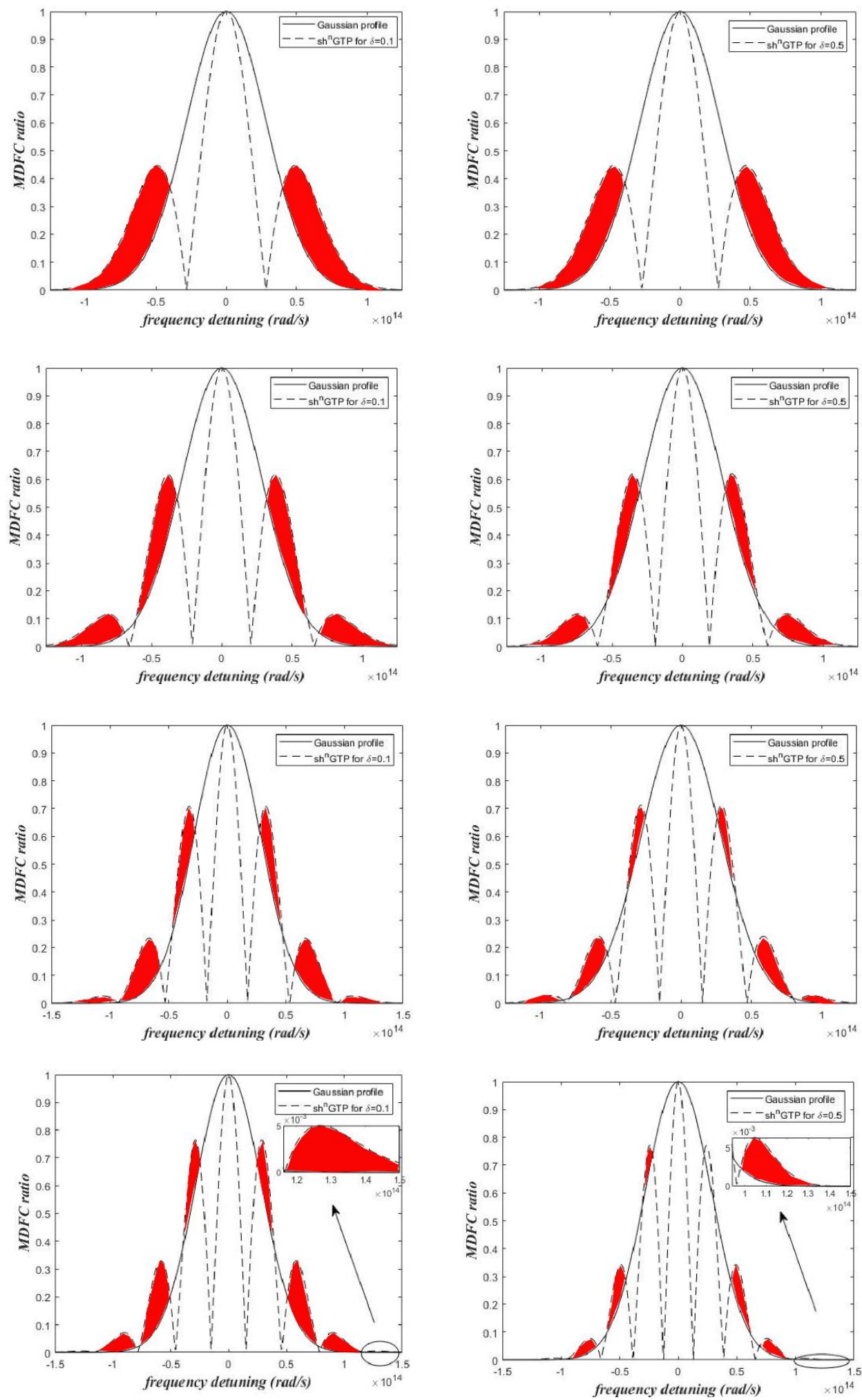

Figure 5: The MDFC ratio as a function of the frequency detuning with different skew parameters $\delta$ and for a fixed value of the pulse duration set as $\tau=50 f s$ and (a) $n=1,(\mathbf{b}) n=2$, (c) $n=3$ and (d) $n=4$. 
In Fig. 6, the MDFC ratio is depicted as a function of the pulse duration and the frequency detuning. It's seen from these plots that the MDFC ratio increases from 0 to 1 as the color changes from blue to yellow (see the rectangular bar).

$n=1$
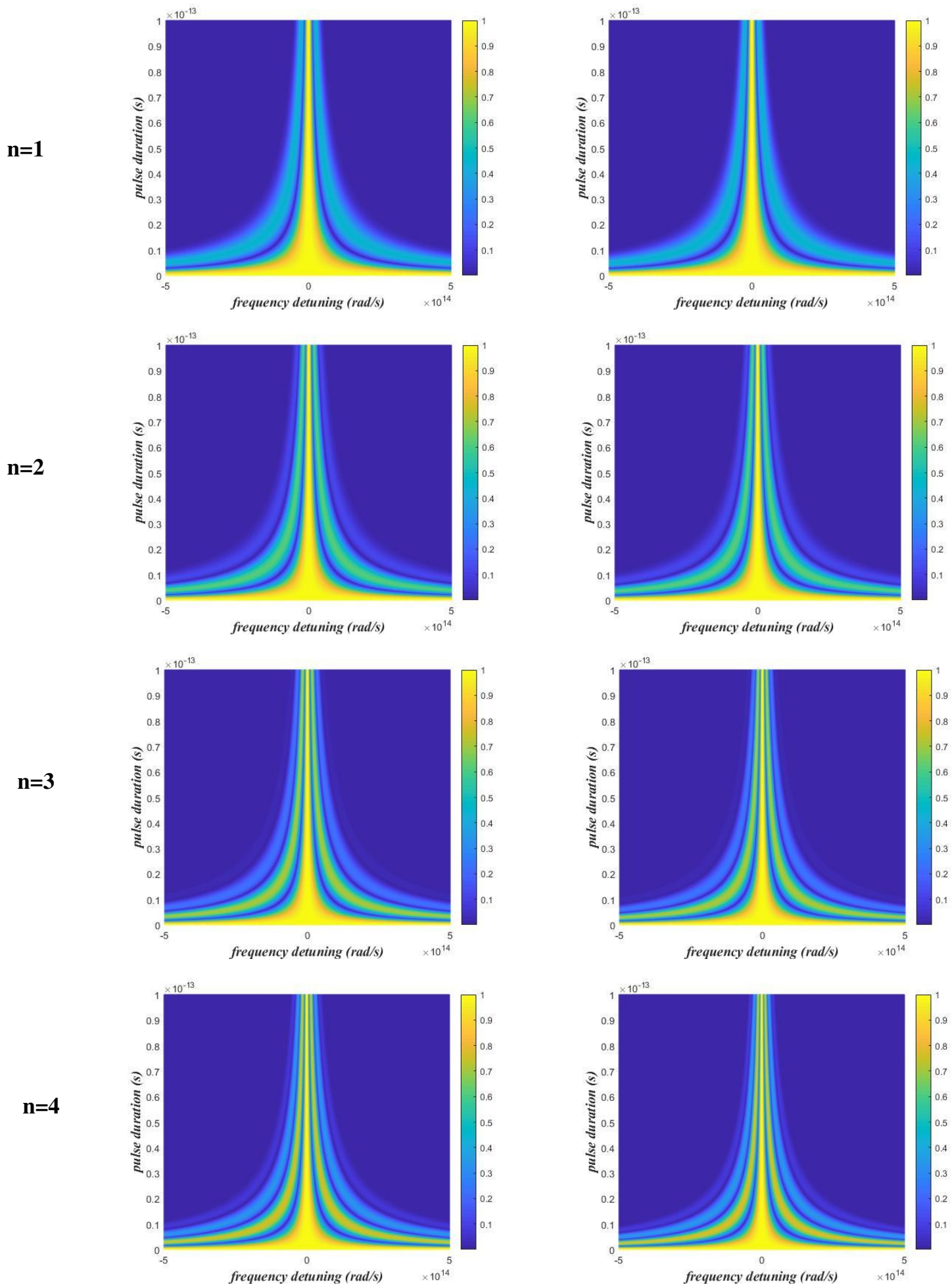

(a)

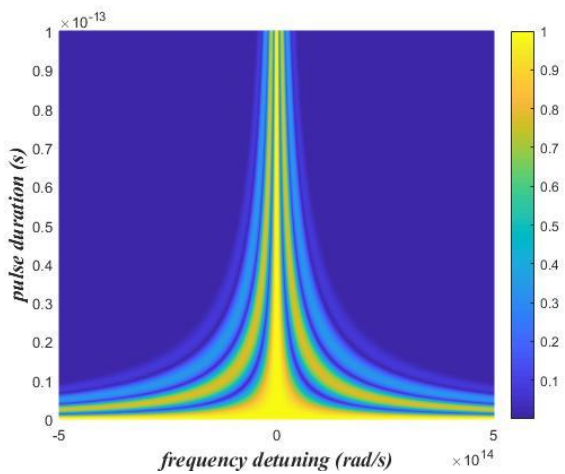

(b)

Figure 6: the MDFC ratio as a function of the pulse duration and the frequency detuning of the $\operatorname{sh}^{\mathrm{n}} \mathrm{GTP}$ for: (a) $\delta=0.1$ and (b) $\delta=0.5$. 
We note that for a particular pulse duration, the MDFC ratio progressively decreases as the frequency detuning increases. After this duration, a thin strip is formed when the MDFC ratio stays zero. As $\Omega$ increases further, the MDFC ratio starts to rise and progressively decreases to 0 , which gives rise to two null regions. We observe that by increasing the beam order $\mathrm{n}$ the new thin strips appear. Hence, the MDFC ratio of $\operatorname{sh}^{\mathrm{n}} \mathrm{GTP}$ presents discontinuities compared to that one of the Gaussian profile, due to the formation of null region (MDCD ratio $=0$ ). The discontinuities increase with the increase of the beam order and a better quality of the hologram can be obtained for this beam profile. We deduce that the $\operatorname{sh}^{\mathrm{n}} \mathrm{GTP}$ is more suitable for obtaining the holograms with good quality.

\subsection{Case of $\operatorname{ch}^{\mathrm{n}} \mathrm{GTP}$}

In this section, we treat the case of $\operatorname{ch}^{\mathrm{n}} \mathrm{GTP}$. We illustrate firstly in Fig. 7, the graphical representations of the temporal profile for this beam family are given for three values of the controlled parameter $\delta$ and for two values of the beam order $n$. We find the Gaussian profile when $\delta=0$ and as the controlled parameter $\delta$ increases and reaches a value 1.2, we observe the appearance of two small lobes, which grow with the increase of $\delta$.

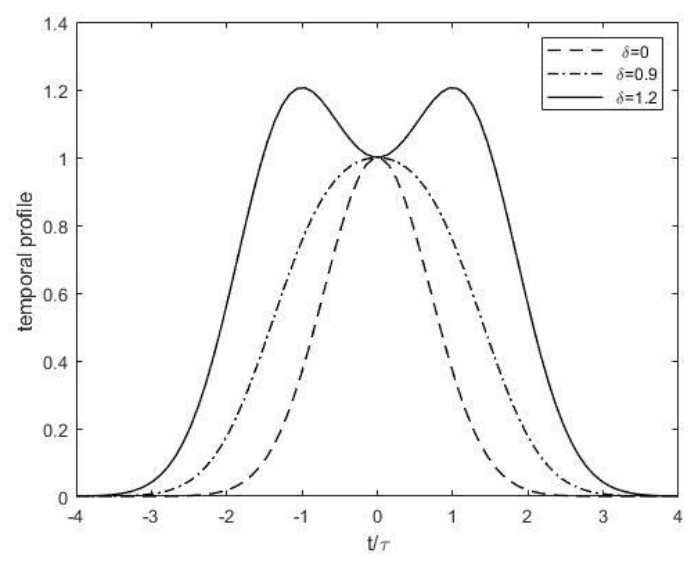

(a)

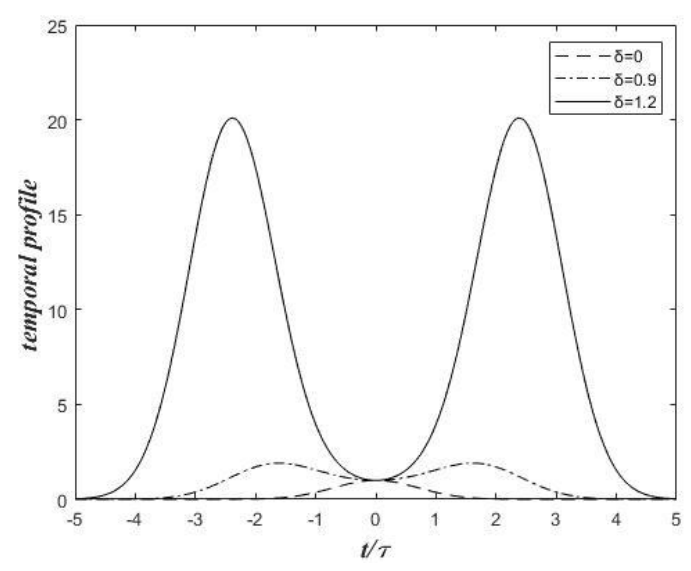

(b)

Figure 7: Temporal profile of $\operatorname{ch}^{\mathrm{n}} \mathrm{GTP}$ for different skew parameters $\delta$ and for: (a) $n=1$ and (b) $n=2$.

We present in Fig. 8 the variation of the modulation depth with the frequency detuning $\Omega$ for three values of the skew parameter $\delta$ of $\operatorname{ch}^{\mathrm{n}}$ GTP having 50 femtoseconds duration. We observe the appearance of new side lobes with the increase of the beam order $n$ and the width of the lobes widens with the decrease of the skew parameter $\delta$. 


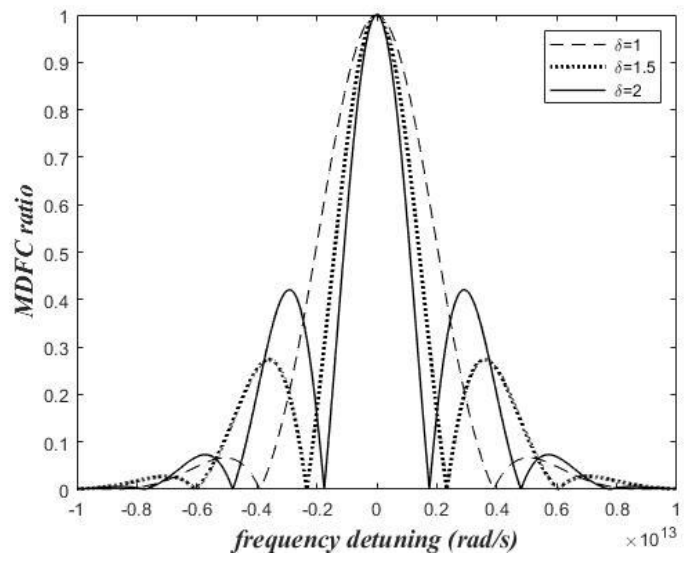

(a)

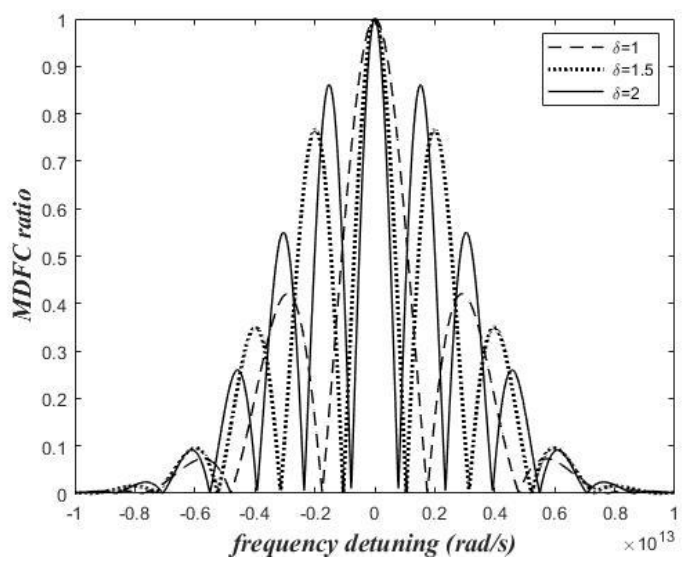

(b)

Figure 8: The MDFC ratio of $\operatorname{ch}^{\mathrm{n}} \mathrm{GTP}$ as a function of the frequency detuning $\Omega$ for: (a) $n=1$ and (b) $n=2$.

Fig. 9 displays the MDFC ratio as a function of the frequency detuning $\Omega$ with three values of the pulses duration $\tau$. It's seen that the total number of secondary lobes starts to increase with the increase of the beam order $n$ and the width of the lobes grow with the decrease of the pulse duration $\tau$.

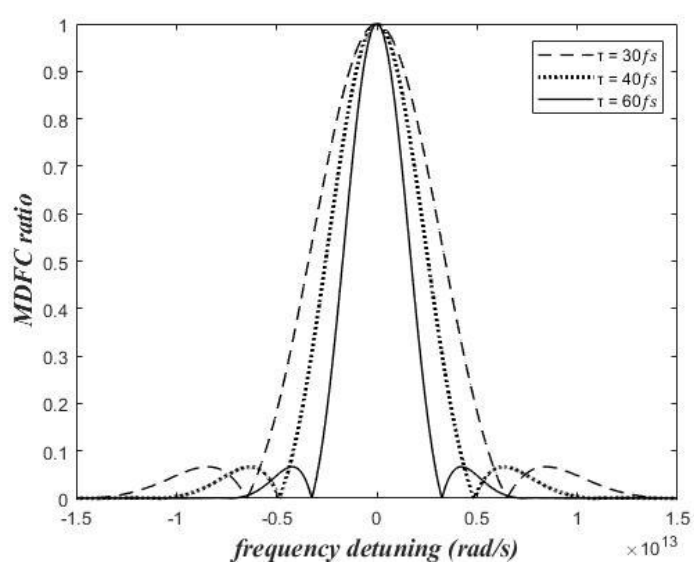

(a)

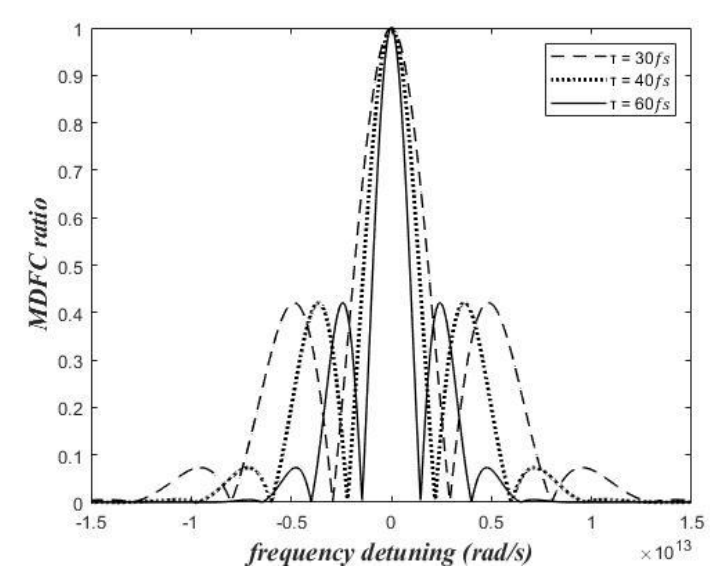

(b)

Figure 9: The MDFC ratio of $\operatorname{ch}^{\mathrm{n}} \mathrm{GTP}$ as a function of the frequency detuning $\Omega$ for: (a) $n=1$ and (b) $n=2$.

Basing on Eq. (43), we present in Fig. 10 the MDFC ratio as a function of the frequency detuning $\Omega$ with different skew parameters $\delta$, for a fixed value of the pulse duration set as $\tau=$ $50 f s$ and for different values of $\mathrm{n}(1,2,3$, and 4). One can easily see from these plots that the $\operatorname{ch}^{\mathrm{n}}$ GTP yields the Gaussian profile when $\delta=0$, and by varying the parameter $\delta$ we see the appearance of side lobes. For example, when $n=2$ (Fig. 10 (b)) for $\delta=1$, we obtain two lobes and when $\delta=1.3$ another lobe that is added. 
(a)
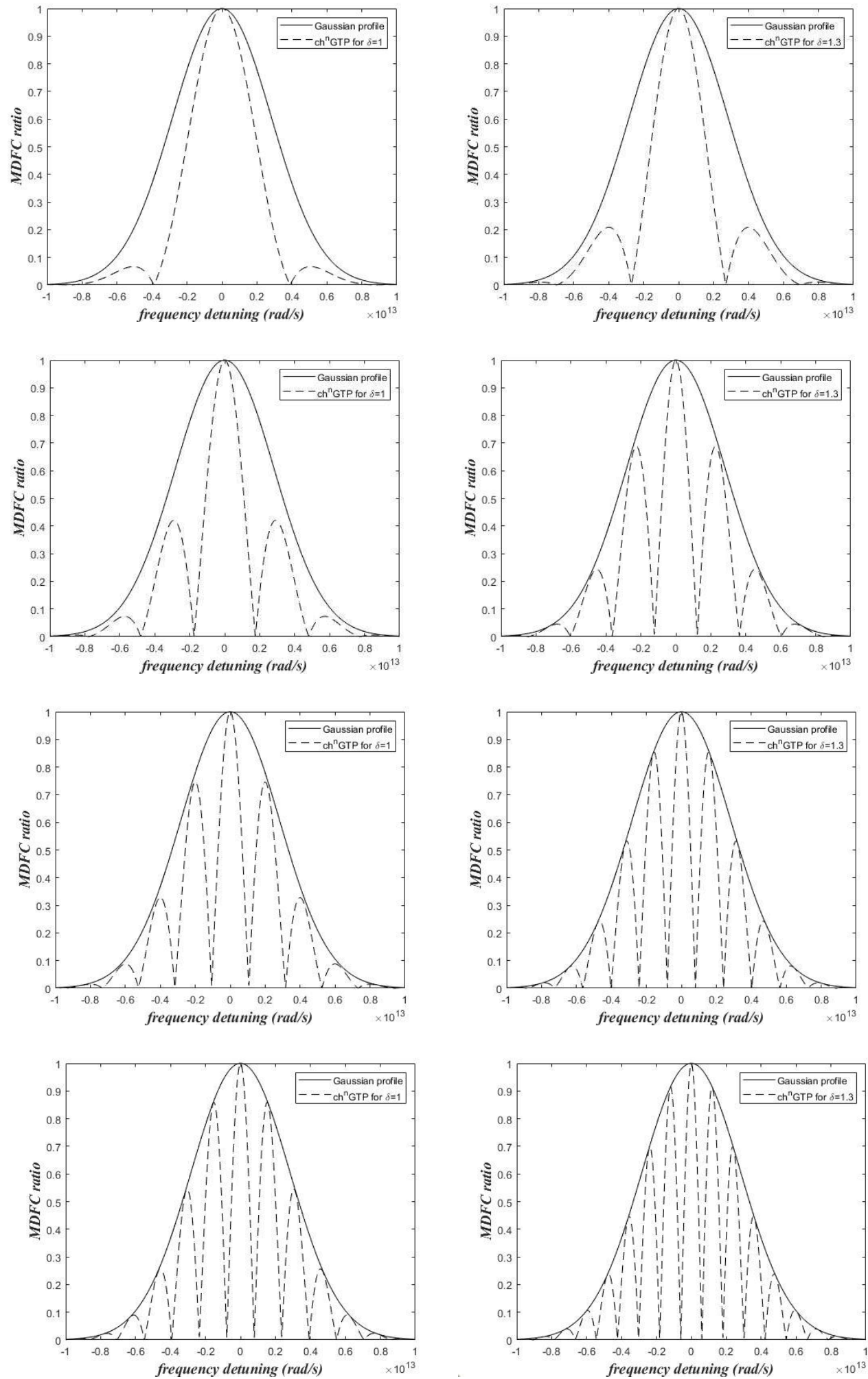

Figure 10: The MDFC ratio of $\operatorname{ch}^{\mathrm{n}} \mathrm{GTP}$ and Gaussian profile as a function of the frequency detuning for $\tau=50 \mathrm{fs}$ and (a) $\mathrm{n}=1,(\mathbf{b}) \mathrm{n}=2$, (c) $\mathrm{n}=3$ and (d) $\mathrm{n}=4$.

The plots in Fig. 11 depict the MDFC ratio as a function of the pulse duration and the frequency detuning for $\operatorname{ch}^{\mathrm{n}} \mathrm{GTP}$ for two values of $\delta$. 

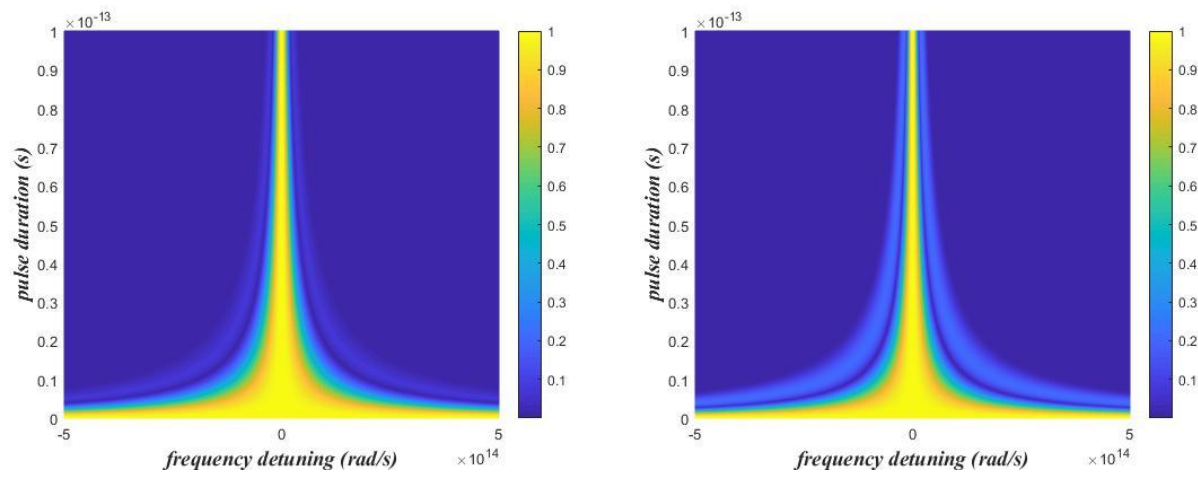

$\mathbf{n}=\mathbf{1}$
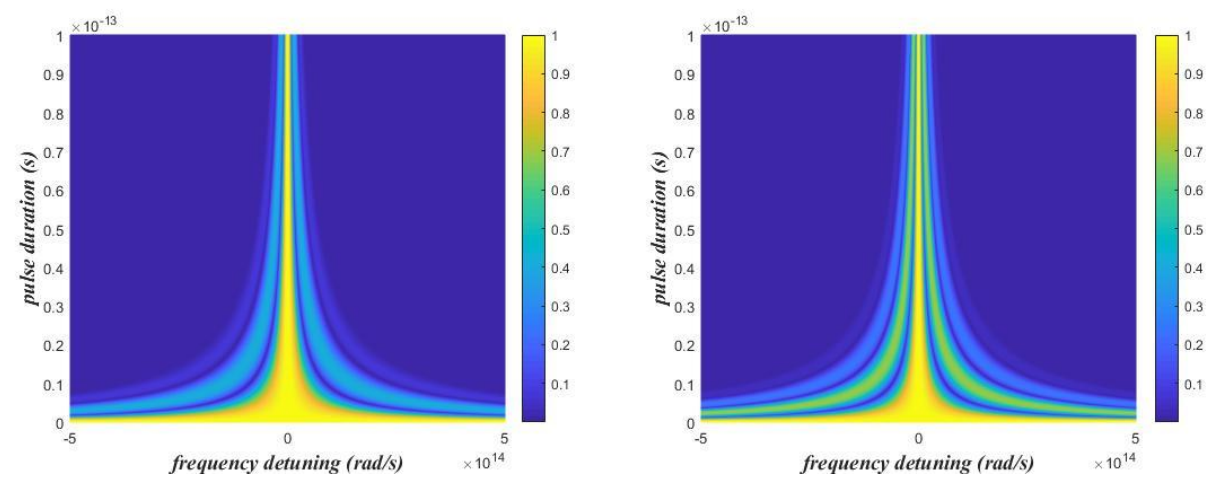

$\mathbf{n}=\mathbf{2}$
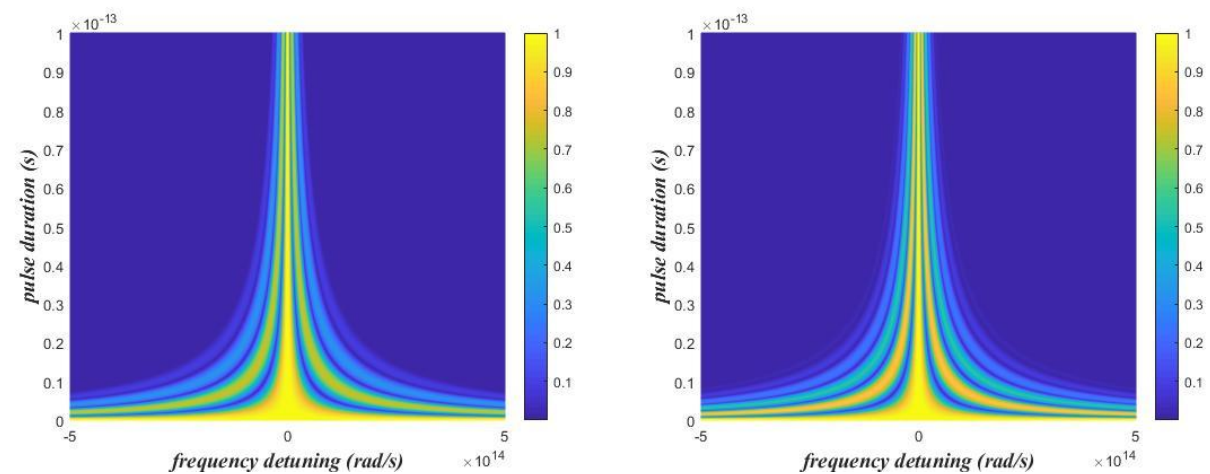

$\mathbf{n}=\mathbf{3}$
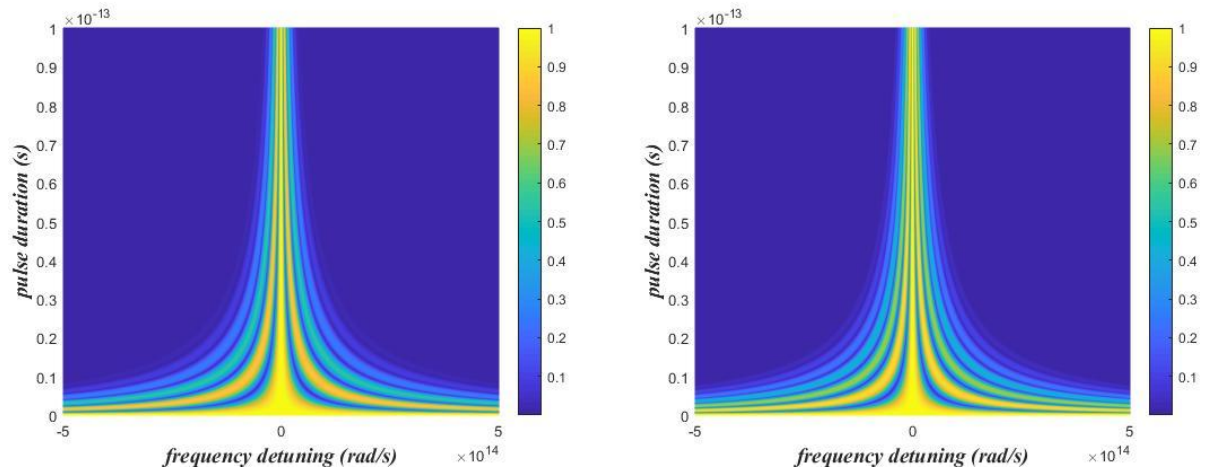

$n=4$

(a)

(b)

Figure 11: The MDFC ratio of $\mathrm{ch}^{\mathrm{n}} \mathrm{GTP}$

as a function of the pulse duration and the frequency detuning for: (a) $\delta=1$ and (b) $\delta=1.3$. 
It's observed from this figure that the decrease from 1 to 0 of the MDFC ratio value as the color becomes darker (see the rectangular bar). Contrary to the Gaussian profile, the $\operatorname{ch}^{\mathrm{n}} \mathrm{GTP}$ is characterized by a MDFC ratio which progressively decreases from 1 (at $\Omega=0$ ) to 0 as the frequency detuning is increased (see Fig. 11) and then a thin strip is formed while the MDFC ratio keeps null. In the end and as $\Omega$ increases further, the MDFC ratio begins to enhance and gradually decreases to zero, which results the appearance of two null regions favoring the formation of the holograms.

\section{Conclusion}

In this paper, we have investigated the process of interference and holography with two laser beams with different amplitudes and different frequencies. We have evaluated in detail the analytical expression of the modulation depth in terms of the fringe contrast, the frequency detuning and the controller parameter of the Higher-order sh- and ch-Gaussian temporal profiles. Based on our main results of the profile evaluated modulation depth, we conclude that the hollow laser beam with dark spot, contrary to Gaussian profile, gives a new area where good quality holograms can be produced for a higher frequency detuning and we showed that the total number of side lobes is proportional to the order of the considered profile. For the second profile family, $\mathrm{ch}^{\mathrm{n}} \mathrm{GTP}$ is favorable to form a good quality hologram. In this later case, we showed that the null regions facilitating the formation of holograms which is more suited to obtaining holograms of good quality. Not only this, our work helps to know the exact combination of the pulse duration and the frequency detuning for the larger modulation depth to fringe contrast ratio gets to the best quality holograms possible. The present work is seeks to present in a plain way that $\operatorname{sh}^{\mathrm{n}} \mathrm{GTP}$ is better than the Gaussian lasers for holography, particularly for higher frequency differences. 


\section{References}

[1] H. Ito, T. Nakata, K. Sakaki, M. Ohtsu, K.I. Lee, W. Jhe, "Laser spectroscopy of atoms guided by evanescent waves in micron-sized hollow optical fibers," Phys. Rev. Lett. 76 (1996) 4500-4503. [2] J. Yin, W. Gao, Y. Zhu, "Generation of dark hollow beams and their applications," Progress in Optics, 45 (2003) 119-204.

[3] T. Kuga, Y. Torii, N. Shiokawa, T. Hirano, Y. Shimizu, H. Sasada, "Novel optical trap of atoms with a doughnut beam,” Phys. Rev. Lett. 78 (1997) 4713-4716.

[4] Y.B. Ovchinnikov, I. Manek, R. Grimm, "Surface trap for cs atoms based on evanescentwave cooling," Phys. Rev. Lett. 79 (1997) 2225-2228.

[5] F. Boufalah, L. Dalil-Essakali and A. Belafhal, "Transformation of a generalized BesselLaguerre-Gaussian beam by a paraxial ABCD optical system", Opt. Elec., 51 (2019) 1-15.

[6] G. Zhou. J. Zheng, "Beam propagation of a higher-order cosh-Gaussian beam", Opt. Laser Technol., 41 (2009) 202-208.

[7] N. Nossir, L. Dalil-Essakali, A Belafhal, "Propagation analysis of some doughnut lasers beams through a paraxial ABCD optical system", Opt. Quant. Electron, 52 (2020) 1-16.

[8] A. Belafhal and F. Saad, "Conversion of circular beams by a spiral phase plate: Generation of Generalized Humbert beams", Optik, 138 (2017) 516-528.

[9] Q. Sun, K. Zhou, G. Fang, G. Zhang, Z. Liu, S. Liu, Hollow sinh-Gaussian beams and their paraxial properties, Opt. Exp, 20 (2012) 9682-9691.

[10] J. Gu, Z. Mei, X. Li, Propagation properties of controllable dark-hollow beams through an annular apertured, misaligned optical system, Optik, 120 (2009) 379-383.

[11] X. Wang, M.G, Littman, "Laser cavity for generation of variable-radius rings of light," Opt. Lett., 18 (1993) 767-768.

[12] H.S. Lee, B.W. Atewart, K. Choi, H. Fenichel, "Holographic nondiverging hollow beam," Phys. Rev. A, 49 (1994) 4922-4927.

[13] R.M. Herman, T.A, Wiggins, "Production and uses of diffractionless beams," J. Opt. Soc. Am. A, 8 (1991) 932-942.

[14] C. Paterson, R. Smith, "Higher-order Bessel waves produced by axicon-type computergenerated holograms,” Opt. Commun., 124 (1996) 121-130.

[15] S. Marksteiner, C.M. Savage, P. Zoller, S, Rolston, "Coherent atomic waveguides from hollow optical fibers,” Quantized atomic motion, Phys. Rev. A, 50 (1994) 2680-2690.

[16] D. Zou, , X. Li, , X. Pang, H. Zheng, Y. Ge, "Propagation properties of hollow sinh-Gaussian beams in quadratic-index medium," Optics Communications, 401(2017) 54-58. 
[17] F. Saad, A. Belafhal, "Propagation properties of Hollow higher-order cosh-Gaussian beams in quadratic index medium and Fractional Fourier transform," Opt Quant Electron 53, 28 (2021) 1-16. [18] C. Rulliere, Femtosecond Laser Pulses: Principal and experiments. Second ed, Springer, Berlin, (1998).

[19] J.C. Diels, W. Rudolph, Ultrashort Laser Pulse Phenomena: Fundamentals, Techniques, and applications on a Femtosecond time scale. Second ed, Academic, San Diego, (1996).

[20] U. Schnars, W. Jueptner, "Direct recording of holograms by a CCD target and numerical reconstruction,” Appl. Opt., 33 (1994) 179-181.

[21] T. Kreis, Handbook of Holographic Interferometry: Optical and Digital Methods. First ed, Wiley-VCH, Weinheim, (2005).

[22] K. Boyer, J. Solem, J. Longworth, A. Borisov, C. Rhodes, "Biomedical three-dimensional holographic microimaging atvisible, ultraviolet and X-ray wavelengths,” Nat. Med., 2 (1996) 939941.

[23] P. Gunter, J.P. Huignard, Photorefractive Materials and Their Applications. Vol. 1 Springer, Berlin, (1988).

[24] Y. Liu, K. Kitamura, S. Takekawa, G. Ravi, M. Nakamura, H. Hatano, T. Yamaji. "Nonvolatile two-color holography in Mndoped near-stoichiometric lithium niobate," Appl. Phys. Lett., 81 (2002) 2686-2688.

[25] G. Zhang, Y. Tomita, "Nonvolatile two-color holography in indium-doped lithium niobate," Appl. Phys. Lett., 77 (2000) 3508-3510.

[26] M. Lee, H. Hatano, S. Tanaka, T. Yamaji, K. Kitamura, S. Takekawa, “Two-color hologram multiplexing from the colored state in stoichiometric LiNbO3: Tb, Fe,” Appl. Phys. Lett., 81 (2002) 4511-4513.

[27] S. Odoulov, A. Shumelyuk, H. Badorreck, S. Nolte, K.M. Voit, M. Imlau, "Interference and holography with femtosecond laser pulses of different colours," Nat. Commun., 6 (2015) 58665873.

[28] L. Malik, A. Escarquel, "Role of the temporal profile of femtosecond lasers of two different colours in holography," EPL, 124 (2018) 64002 (1-6).

[29] L. Malik, A. Escarquel, "Dark hollow lasers may be better candidates for holography," Optics and Laser Technology, 132 (2020) 106485-106491.

[30] I.S. Gradshteyn, I.M. Ryzhik, A. Jeffrey. Table of integrals, series, and products. 7th ed, Academic Press, Amsterdam; Boston, (2007). 


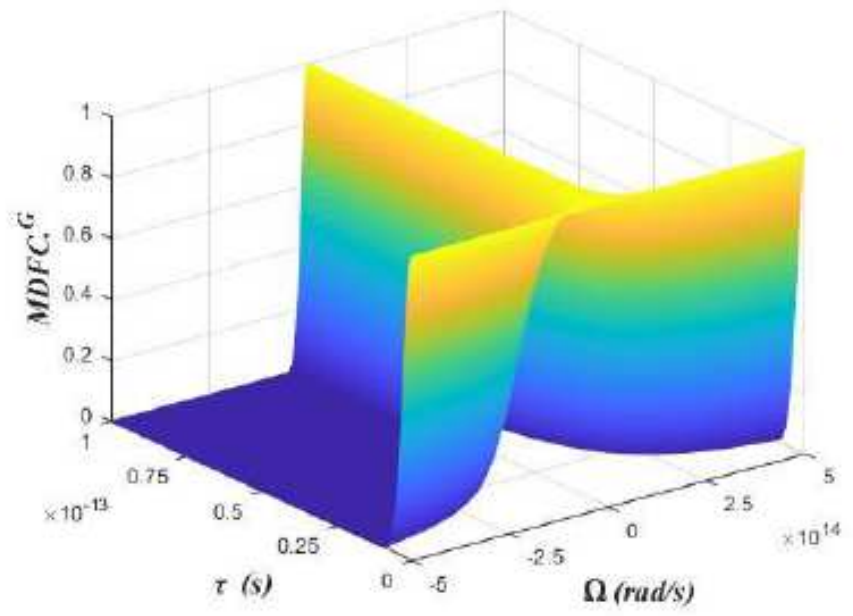

(a)

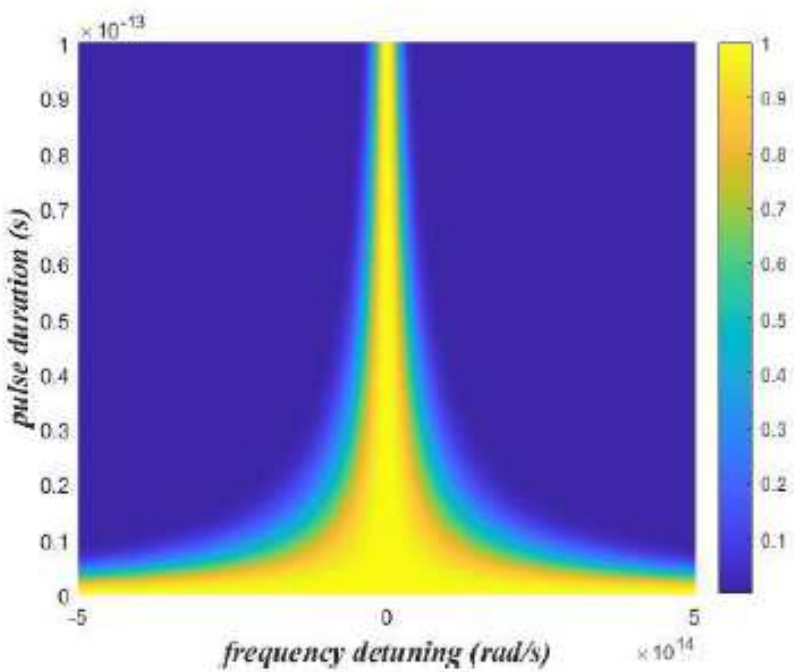

(b)

\section{Figure 1}

The MDFC ratio as a function of the pulse duration $\tau$ and frequency detuning $\Omega$ of the Gaussian pulses.

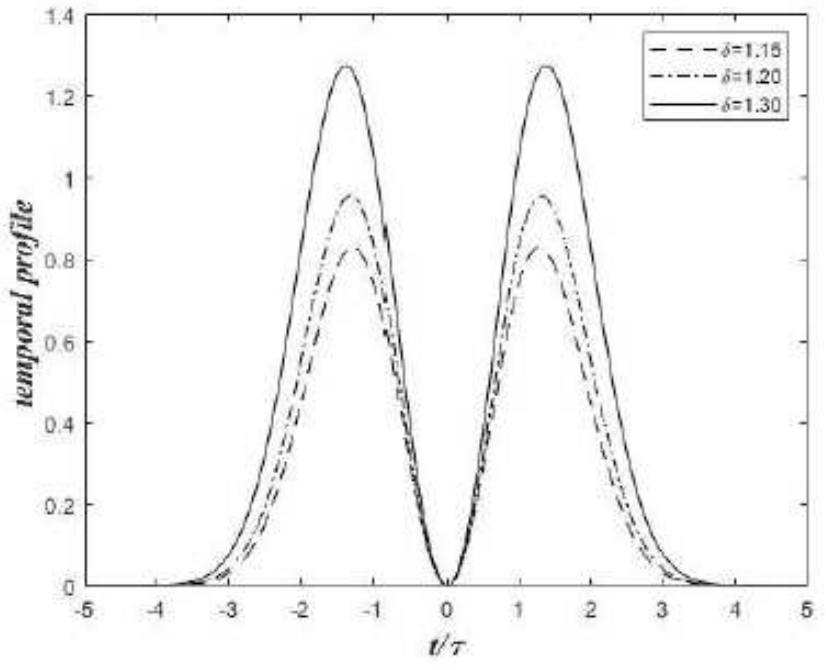

(a)

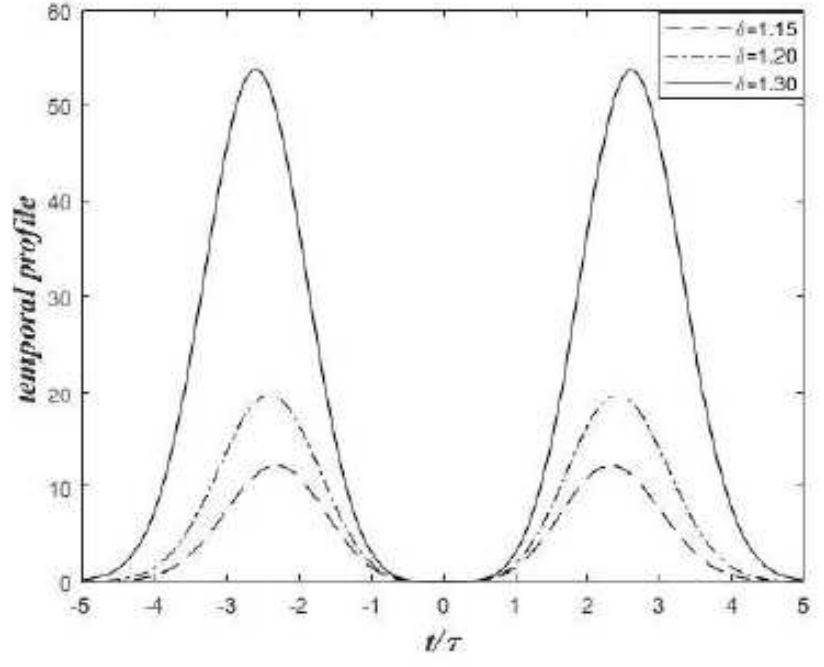

(b)

\section{Figure 2}

Temporal profile of shnGTP for different skew parameters $\delta$ and for: (a) $n=1$ and (b) $n=2$. 


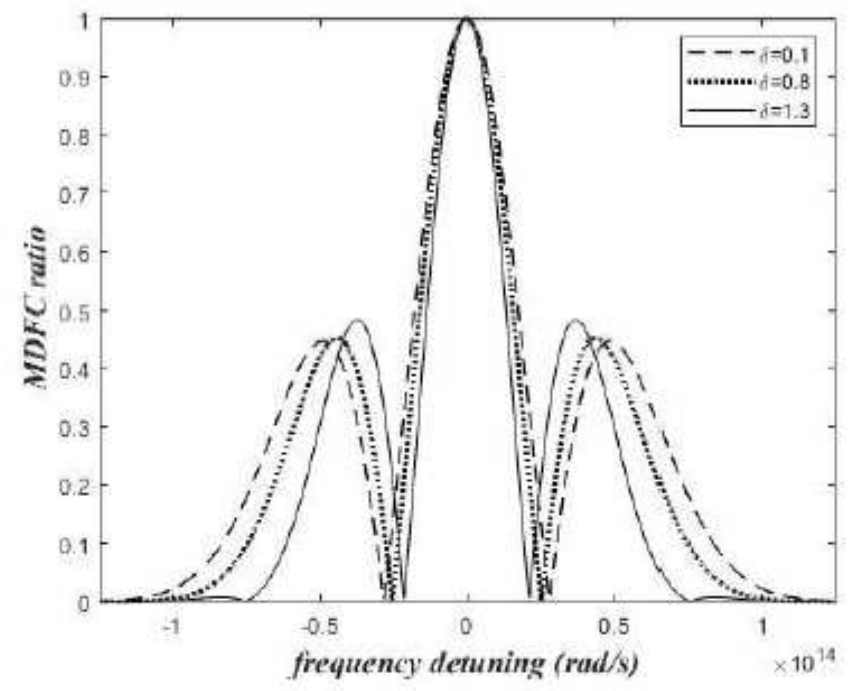

(a)

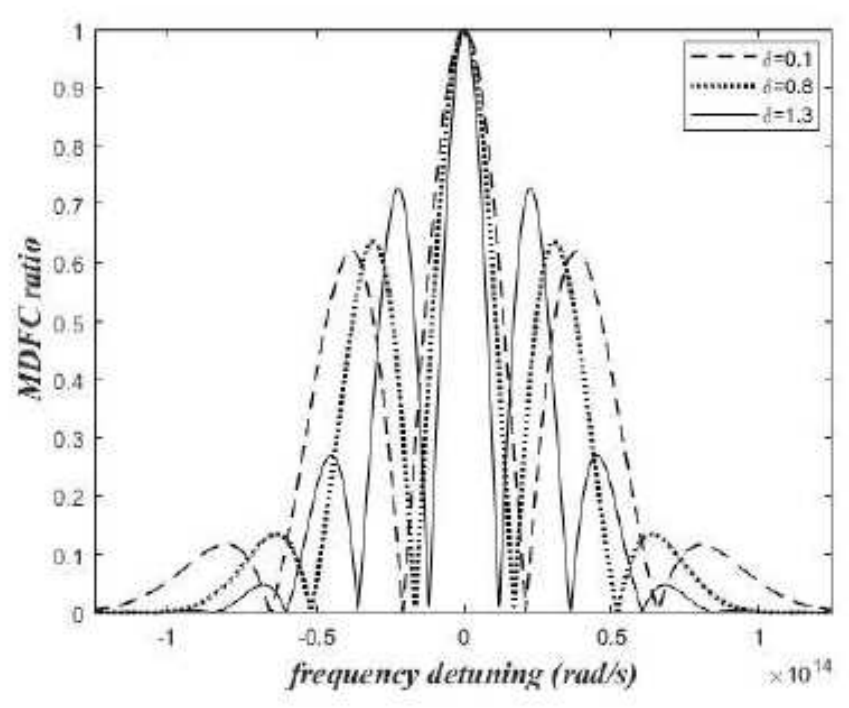

(b)

\section{Figure 3}

Behaviour of the MDFC ratio of shnGTP as a function of the frequency detuning and for a fixed value of the pulse duration set as $\tau=50 \otimes$ and for: (a) $n=1$ and (b) $n=2$.

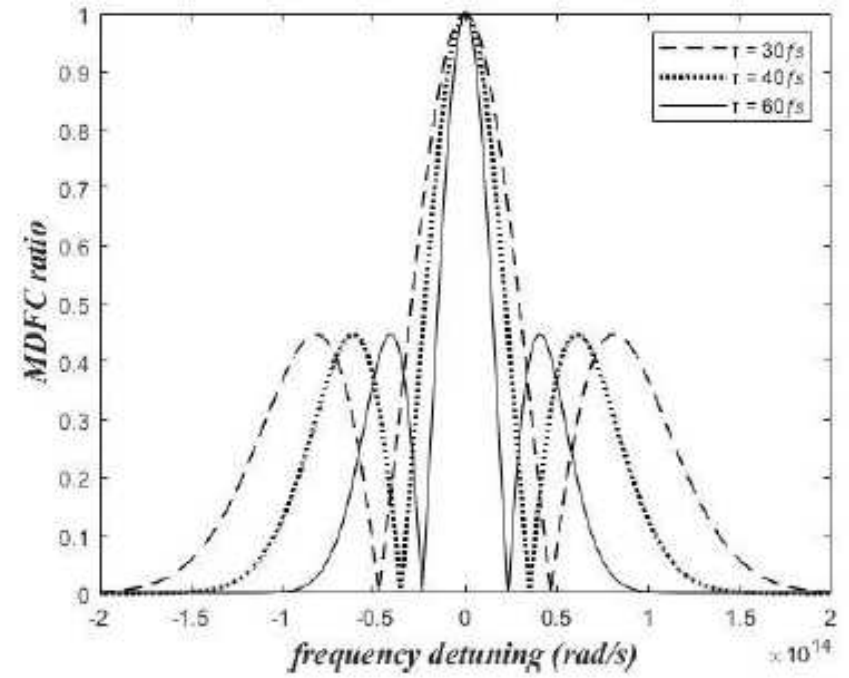

(a)

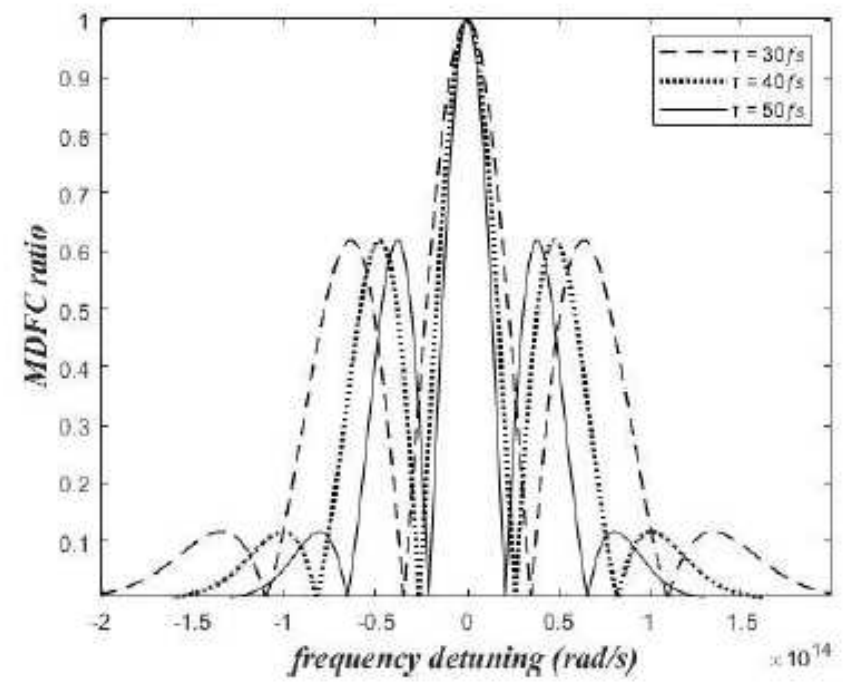

(b)

\section{Figure 4}

Plots of the MDFC ratio of shnGTP with the frequency detuning for: (a) $n=1$ and (b) $n=2$. 
(a)
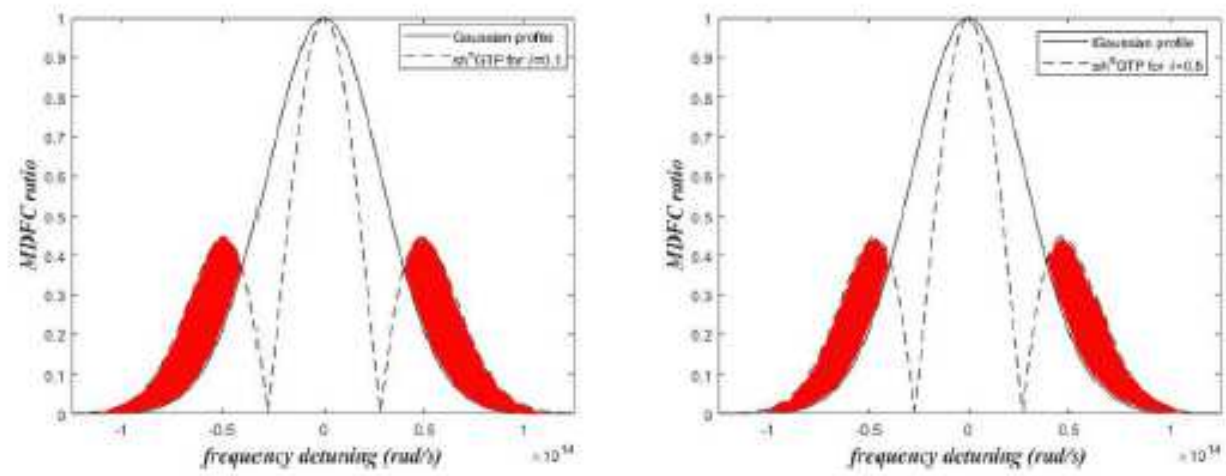

(b)
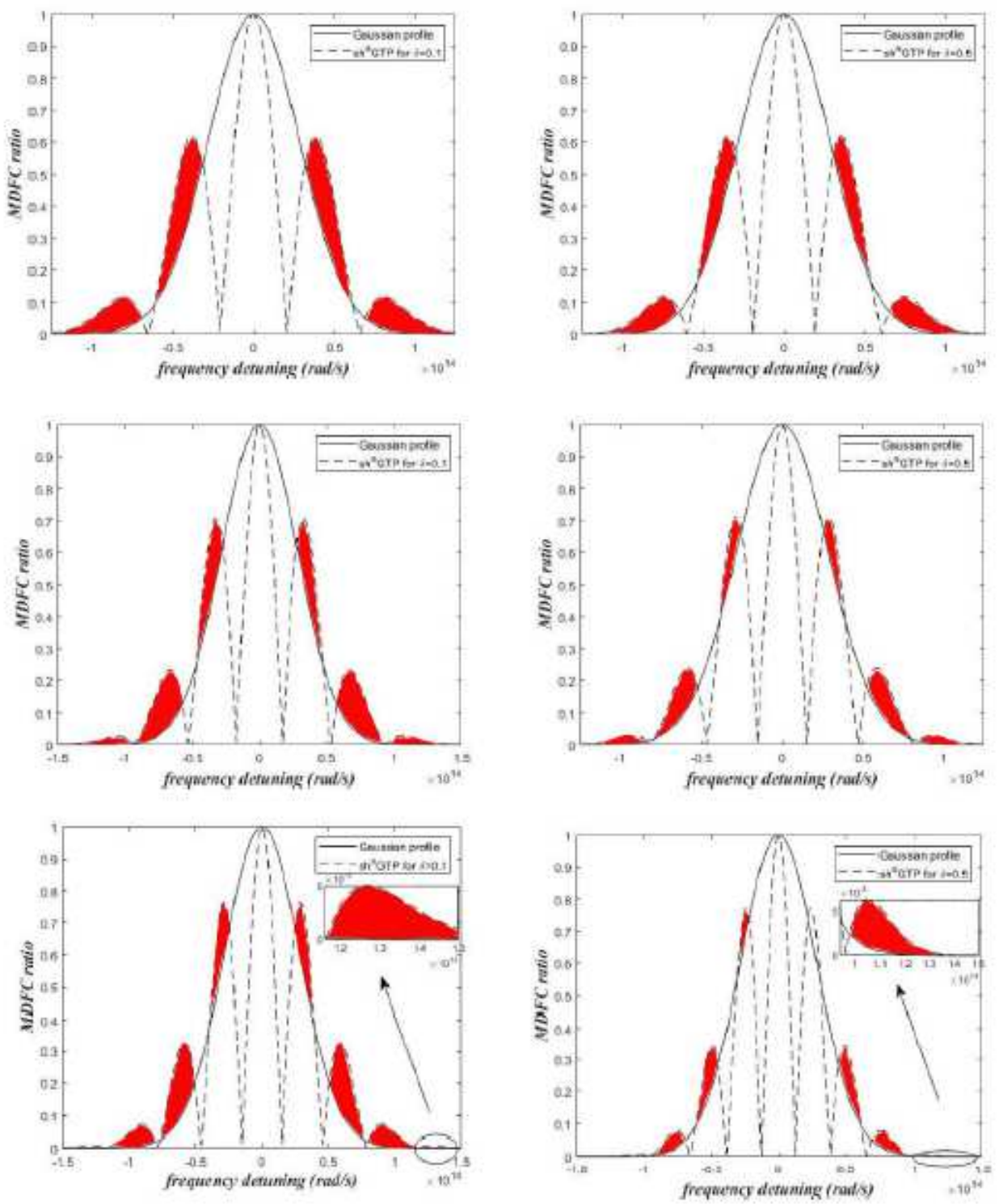

Figure 5

The MDFC ratio as a function of the frequency detuning with different skew parameters $\delta$ and for a fixed value of the pulse duration set as $\tau=50 \mathrm{~B}$ and (a) $n=1$, (b) $n=2$, (c) $n=3$ and (d) $n=4$. 

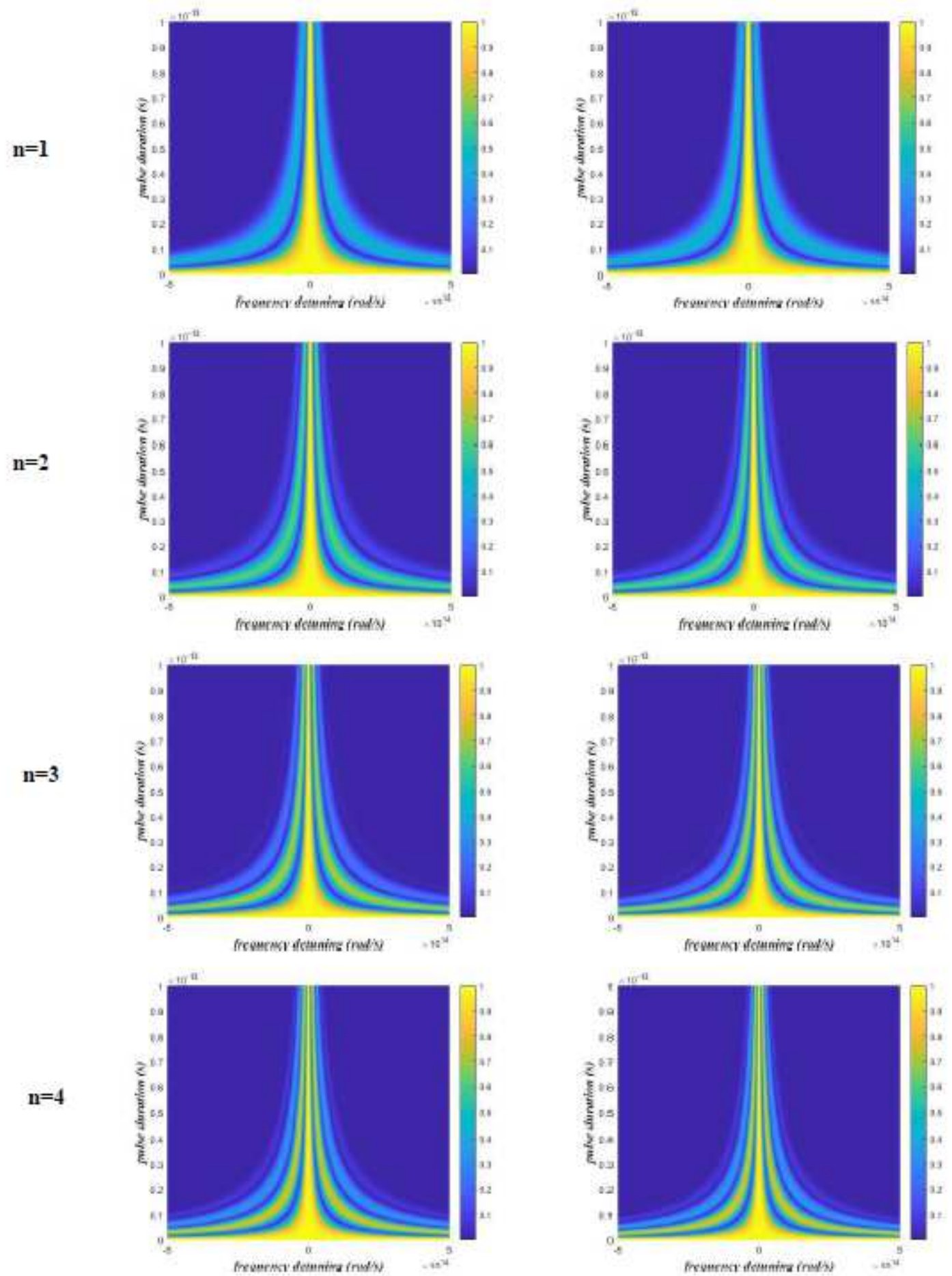

(a)

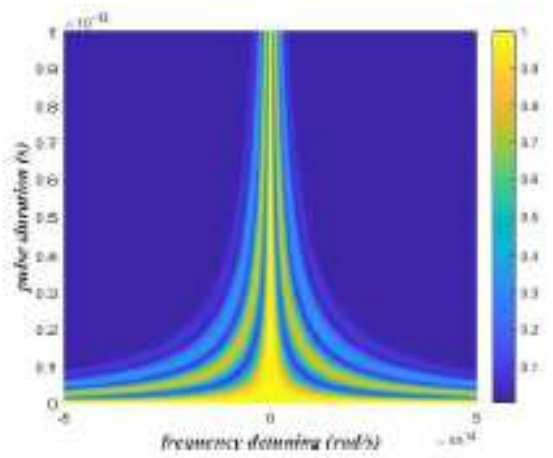

(b)

\section{Figure 6}

: the MDFC ratio as a function of the pulse duration and the frequency detuning of the shnGTP for: (a) $\square=$ 0.1 and $(b) \otimes=0.5$. 


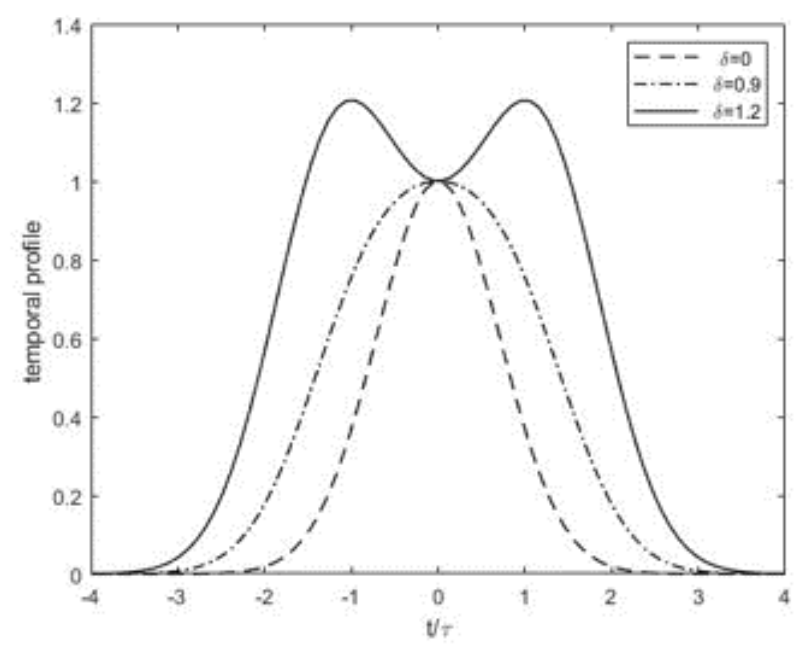

(a)

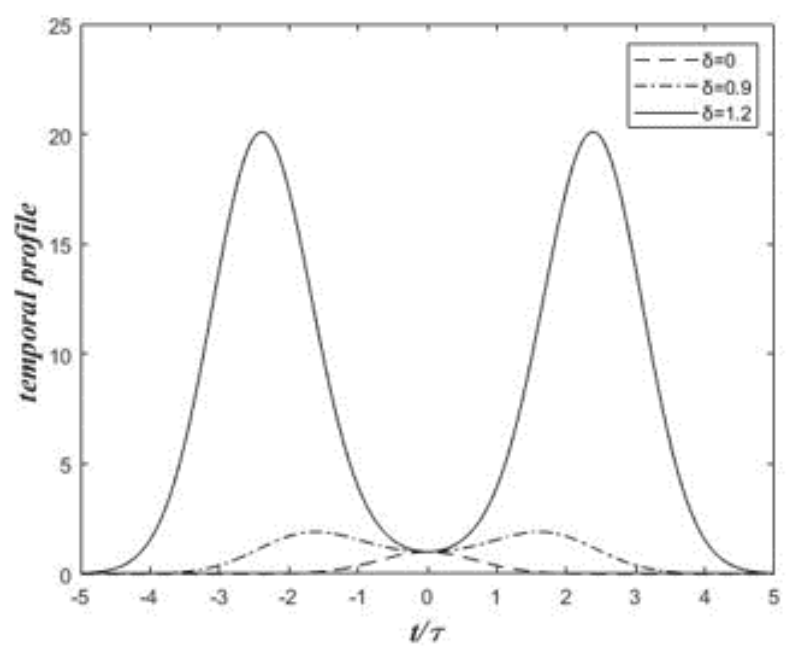

(b)

Figure 7

Temporal profile of chnGTP for different skew parameters $\delta$ and for: (a) $n=1$ and (b) $n=2$.

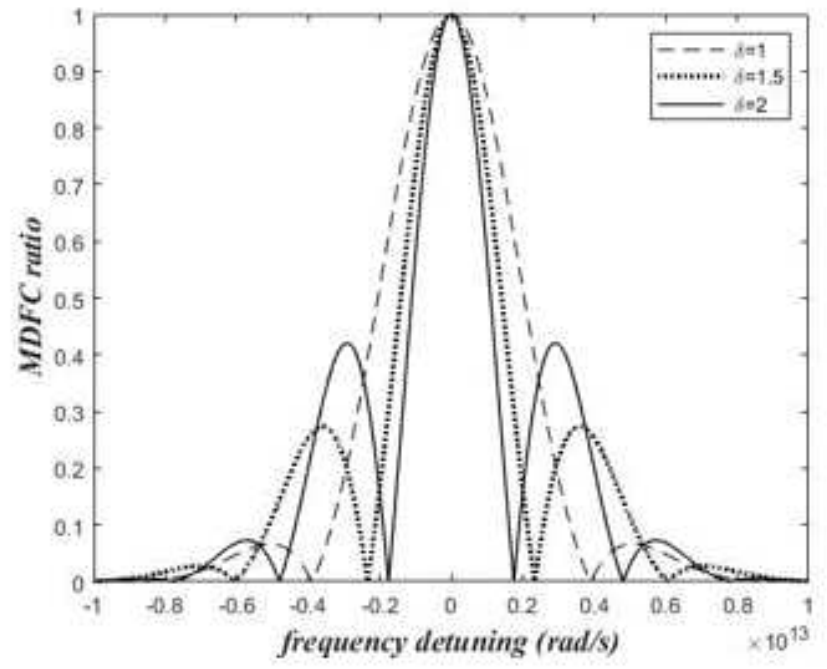

(a)

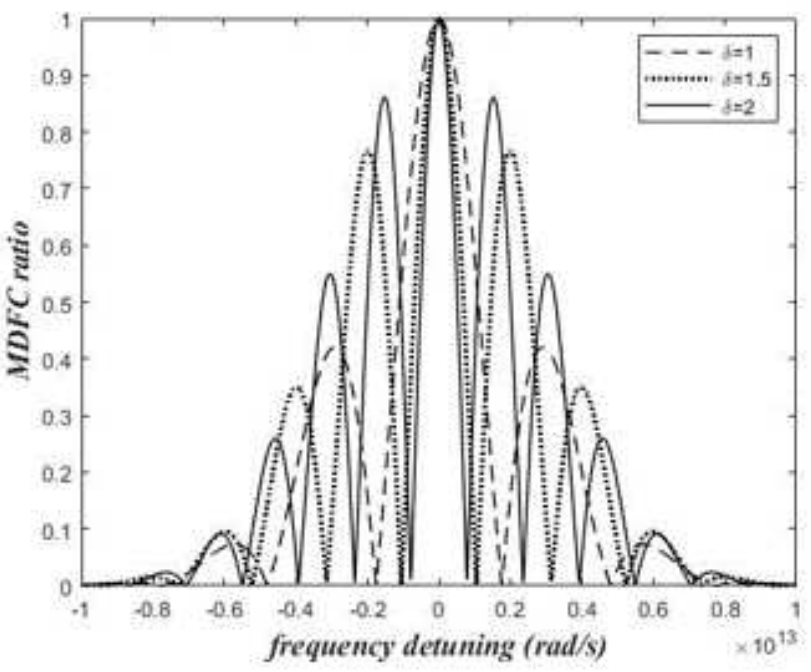

(b)

\section{Figure 8}

The MDFC ratio of chnGTP as a function of the frequency detuning $\Omega$ for: (a) $n=1$ and (b) $n=2$. 


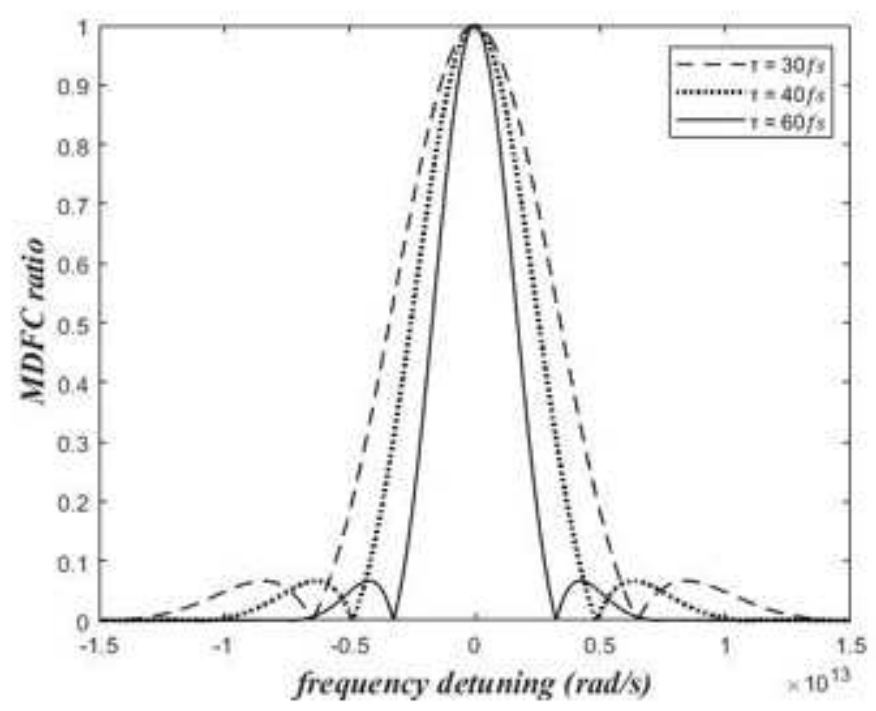

(a)

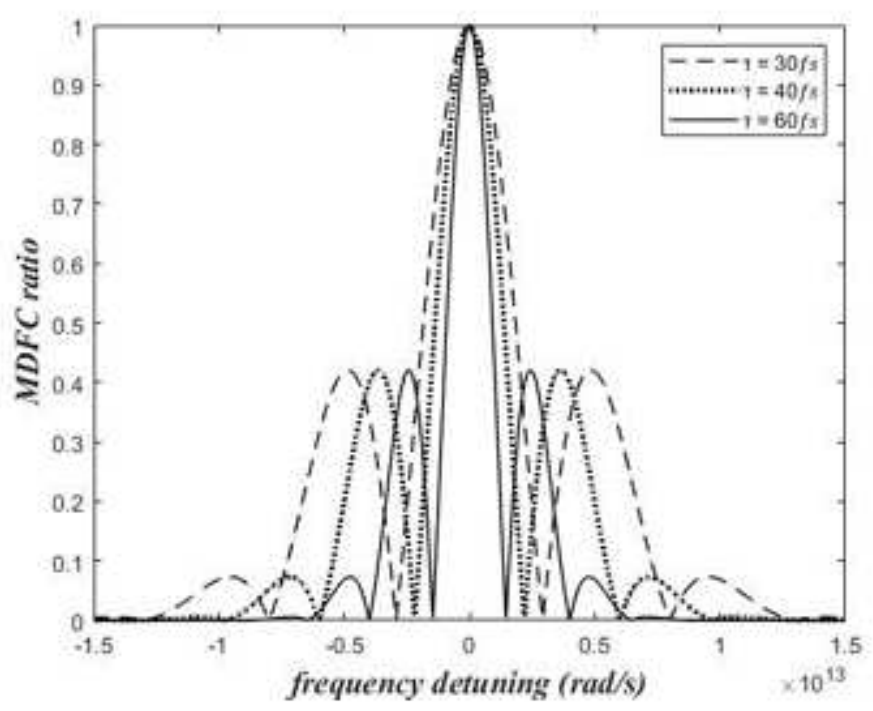

(b)

\section{Figure 9}

The MDFC ratio of chnGTP as a function of the frequency detuning $\Omega$ for: (a) $n=1$ and (b) $n=2$. 
(a)

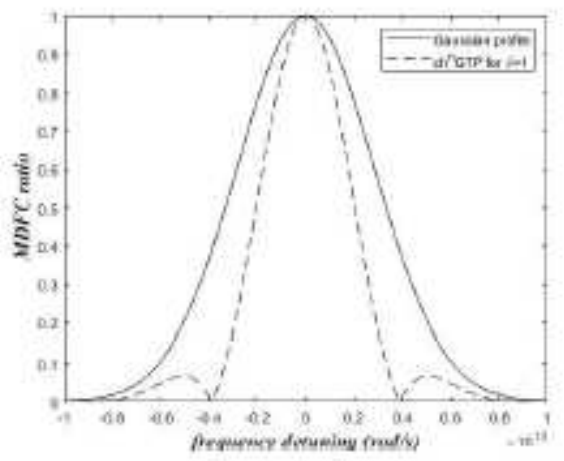

(b)

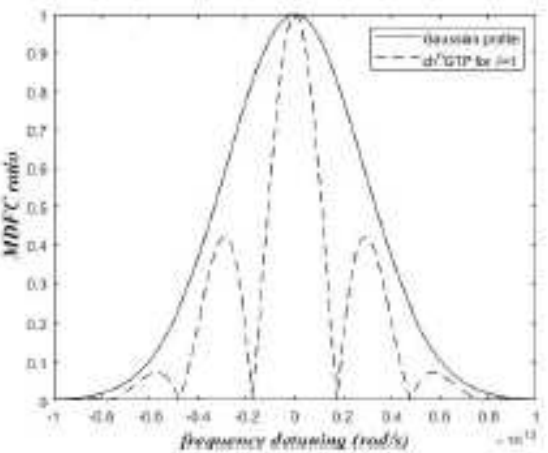

(c)

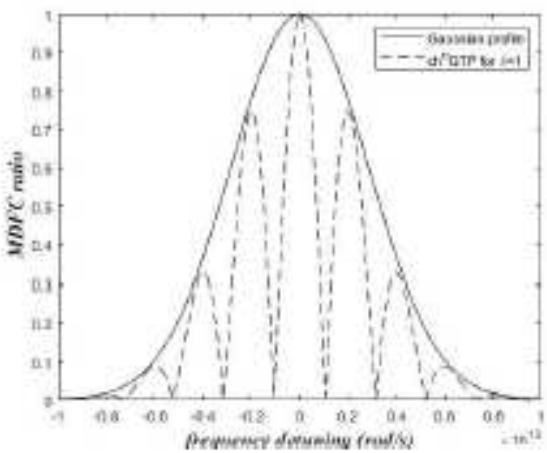

(d)

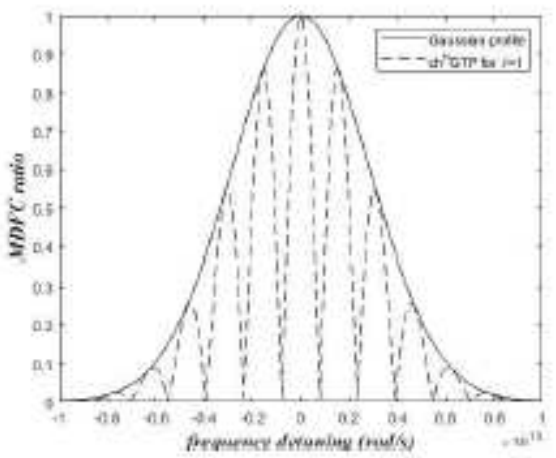

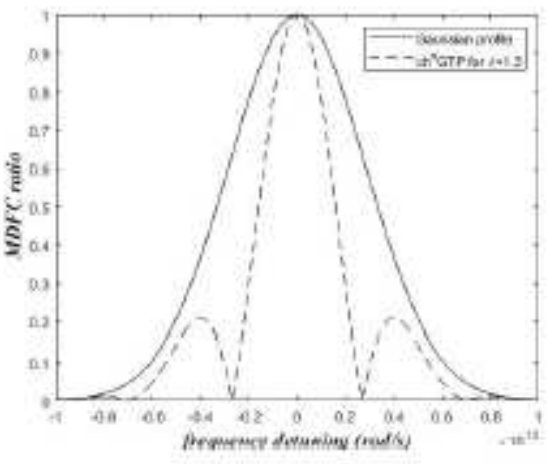
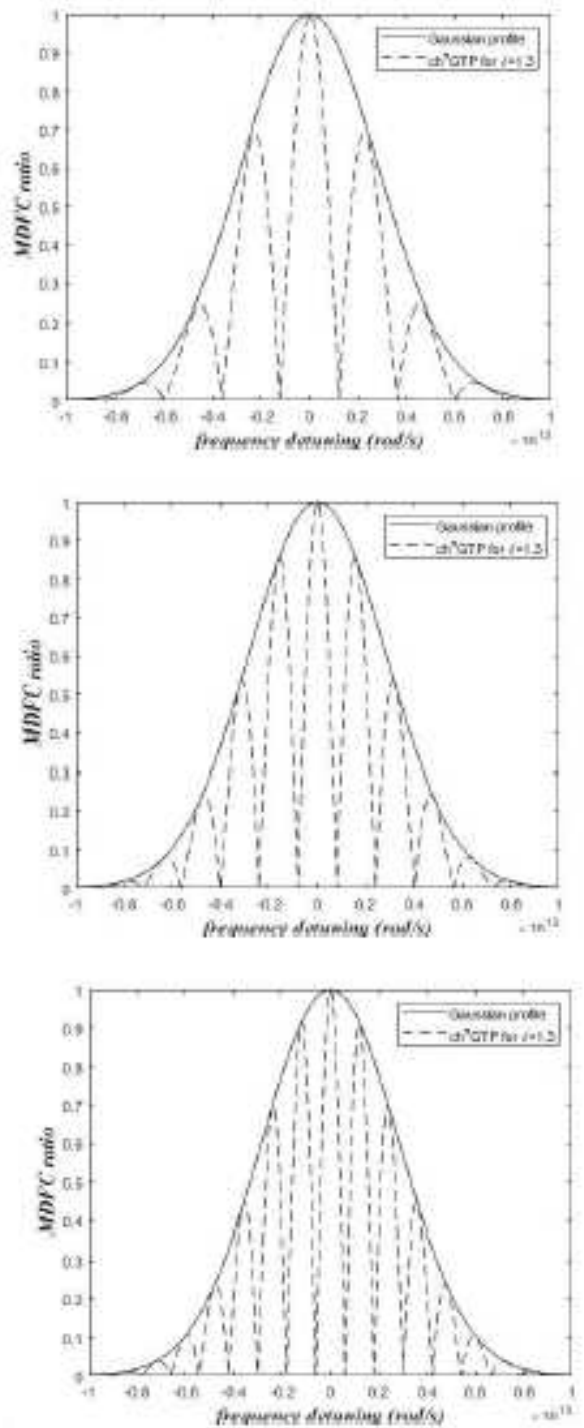

Figure 10

The MDFC ratio of chnGTP and Gaussian profile as a function of the frequency detuning for $\tau=50 \mathrm{BD}$ and (a) $n=1,(b) n=2,(c) n=3$ and (d) $n=4$. 

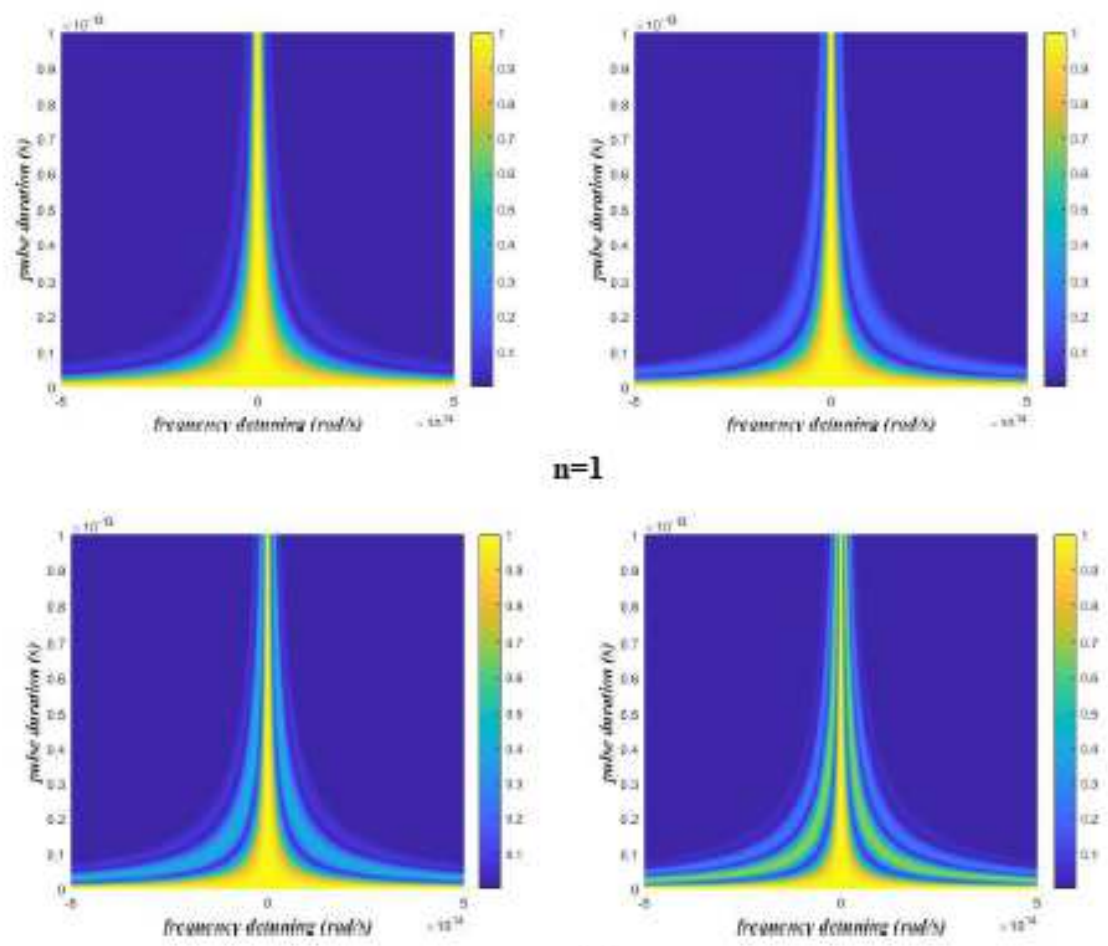

$\mathrm{n}=1$

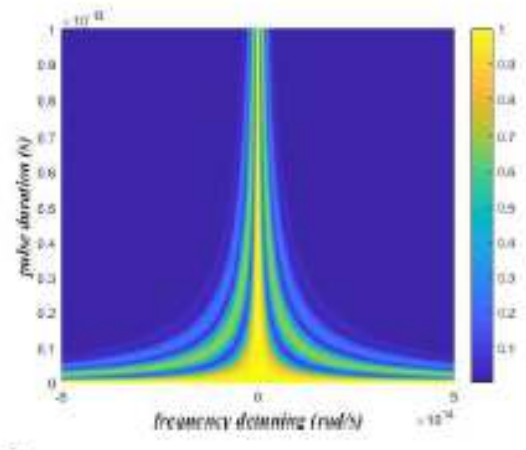

$n=2$
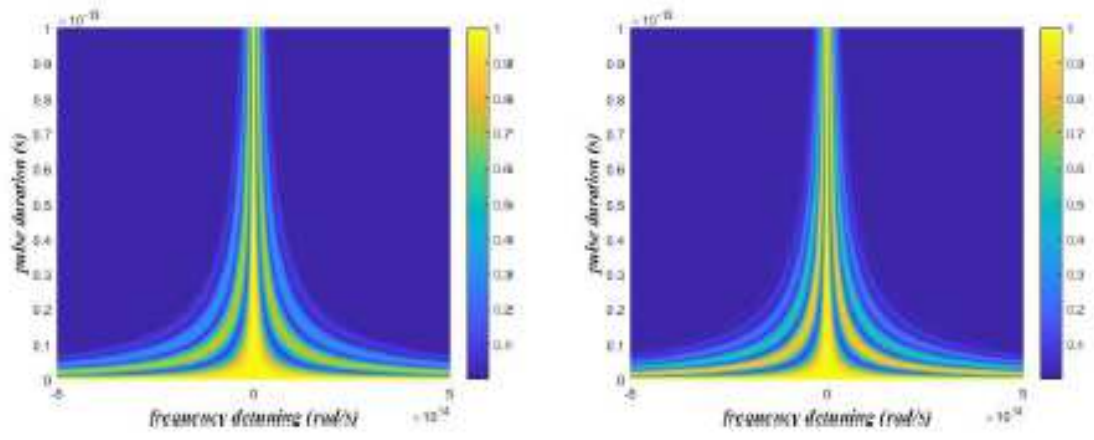

$n=3$
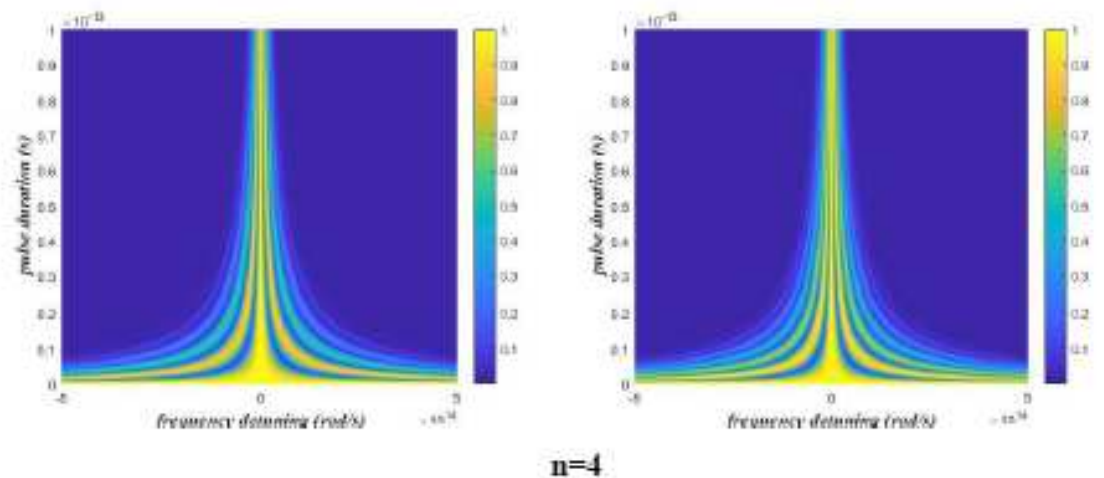

Figure 11

The MDFC ratio of chnGTP as a function of the pulse duration and the frequency detuning for: (a) $\nabla=1$ and (b) $\otimes=1.3$. 